\title{
Some Generalizations of the Pinwheel Tiling*
}

\author{
L. Sadun \\ Mathematics Department, University of Texas, \\ Austin, TX 78712, USA \\ sadun@math.utexas.edu
}

Communicated by Marjorie Senechal

\begin{abstract}
We introduce a new family of nonperiodic tilings, based on a substitution rule that generalizes the pinwheel tiling of Conway and Radin. In each tiling the tiles are similar to a single triangular prototile. In a countable number of cases, the tiles appear in a finite number of sizes and an infinite number of orientations. These tilings generally do not meet full-edge to full-edge, but can be forced through local matching rules. In a countable number of cases, the tiles appear in a finite number of orientations but an infinite number of sizes, all within a set range, while in an uncountable number of cases both the number of sizes and the number of orientations is infinite.
\end{abstract}

\section{Introduction}

We introduce a new family of nonperiodic tilings, indexed by a continuous parameter. In each tiling the tiles are similar to a single triangular prototile. In a countable number of cases, the tiles appear in a finite number of sizes and an infinite number of orientations. In a countable number of cases, the tiles appear in a finite number of orientations but an infinite number of sizes, all within a set range. In one case both the number of sizes and orientations is finite, while in an uncountable number of cases both the number of sizes and the number of orientations is infinite. A piece of a tiling with two sizes and an infinite number of orientations is shown in Fig. 1.

These tilings all arise from a substitution scheme that is quite similar to the pinwheel tiling of Conway and Radin [R1]. In all cases the tilings have the "sibling edge-to-edge"

\footnotetext{
* This research was supported in part by an NSF Mathematical Sciences Postdoctoral Fellowship and Texas ARP Grant 003658-037.
} 


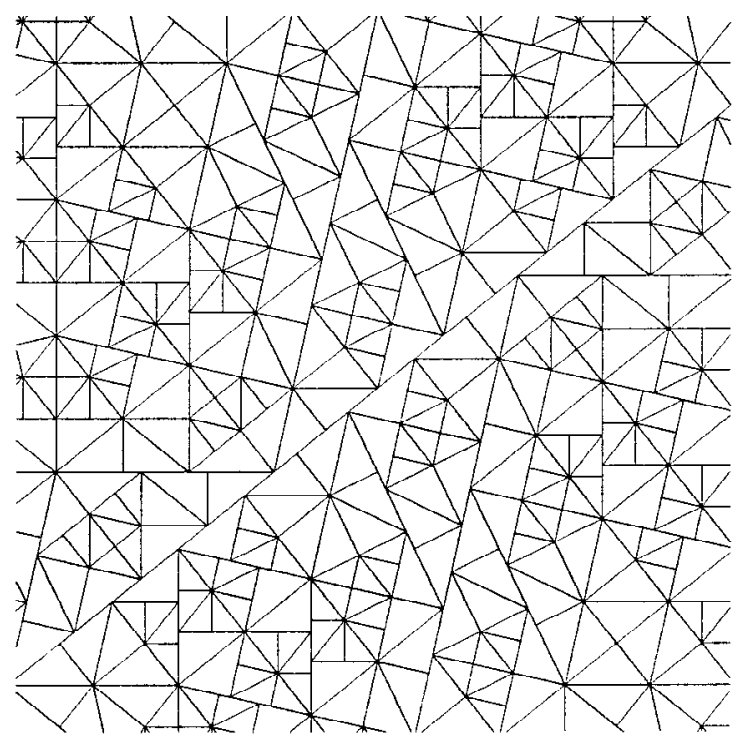

Fig. 1. Part of the tiling Til(1/2).

property, which is to say that two daughter tiles of a single parent tile can only meet full-edge to full-edge. However, in general the tiling is not globally edge-to-edge. Tiles that are not siblings generally do meet in ways that are not full-edge to full-edge.

Goodman-Strauss [G] has recently announced that substitution tilings with a finite number of prototiles, with each prototile appearing in a finite number of sizes, and with the sibling edge-to-edge property, may be associated to local matching rules. These rules are less restrictive than local atlases, and in some cases may allow two adjacent tiles to slide freely along their common edge. However, the rules are restrictive enough to force any tiling to be hierarchical (i.e., generated by the substitution scheme), and hence to be nonperiodic. In a countable number of cases considered in this paper, the hypotheses of Goodman-Strauss's theorem are met.

This is striking, because these examples are definitely not globally edge-to-edge. Indeed, some of these tilings contain infinitely many pairs of tiles with edges that partially overlap. This phenomenon, called "slippage," can be seen in Fig. 1, especially along the long diagonal. The lengths of the overlaps are not the same for all pairs of tiles. In the tiling of Fig. 1, in fact, overlaps occur with an infinite number of distinct lengths. Thus we have the (seemingly) paradoxical situation that Goodman-Strauss's local matching rules force a hierarchical pattern that, in turn, forces an infinite variety of local behavior.

The tilings of this paper also suggest a relaxation of the rules of the tiling dynamical systems game. Prior to Conway and Radin's work, a tiling dynamical system required that all tiles be generated from a finite set of prototiles by translation only, with rotation and reflection not allowed. By those rules, the pinwheel tiling, which involves an infinite number of orientations of a single triangle, uniformly distributed about the circle, was not a tiling! The pinwheel example helped force a reconsideration of the rules, and a 
new consensus has emerged that tiles may be generated from prototiles by Euclidean motions, not just by translations.

The present examples suggest a further extension from the Euclidean group to the conformal group. In almost all cases, these tilings consist of a single prototile appearing in an infinite number of sizes and an infinite number of orientations. Although the distribution of sizes is not constant (which it could not be, as the conformal group has no Haar measure), it is described by a piecewise-constant function.

Such symmetry is related to properties of the spectrum. In the case of the pinwheel, Radin [R2] showed that the statistical rotational invariance of the system results in a spectrum that is rotationally invariant, and hence contains no discrete component. However, Radin's argument did not address the dependence of the spectrum on the radial variable, which corresponds to a length scale. Almost all the examples in this paper have a joint distribution of sizes and orientation that is rotationally invariant, proving (by Radin's argument) that they have continuous spectrum. In addition, in almost all these examples the distribution is absolutely continuous in the size parameter. We conjecture that, in these cases, the spectrum is purely absolutely continuous.

Purists who object to the extension to the conformal group may restrict their attention to the countable and dense set of examples for which only a finite number of sizes appear. They may then take these sizes of the basic triangle as their prototiles, which then generate all tiles via Euclidean motions. We call such examples "rational tilings."

The division of the paper is as follows. In Section 2 we explain the substitution rules and the resulting constructions of the tilings, and classify right triangles according to whether they generate finite numbers of sizes and orientations, finite sizes and infinite orientations, infinite sizes and finite orientations, or infinite sizes and infinite orientations. In Section 3 we examine in detail an example in which tiles appear in two sizes and an infinite number of orientations. We prove that this tiling is sibling edge-to-edge but exhibits slippage; this tiling contains pairs of tiles that meet in an infinite number of distinct ways. In Section 4 we compute the limiting distribution of sizes and orientations in all rational tilings. In Section 5 we compute the statistical distribution of sizes and orientations in irrational tilings. In Section 6 we examine two exceptional rational tilings.

\section{Definitions and Constructions}

As with any substitution scheme, we obtain a tiling of the plane by a succession of subdivisions ("deflations"), rescalings, and repositionings. We start with a single prototile $T$, define a subdivision rule, and let $T_{n}$ be the result of subdividing the basic tile $n$ times. We construct infinite sequences of integers $N_{1}<N_{2}<\cdots$, rescalings $s_{i}$ and Euclidean motions $e_{i}$ such that $e_{i}\left(s_{i}\left(T_{N_{i}}\right)\right)$ is a proper subtiling of $e_{i+1}\left(s_{i+1}\left(T_{N_{i+1}}\right)\right)$. By taking the union of the finite tilings $e_{i}\left(s_{i}\left(T_{N_{i}}\right)\right)$, we obtain a tiling of an infinite region, typically the entire plane.

Our task is complicated by the fact that there is not a straightforward connection between $N_{i}$ and $s_{i}$. (Unlike, say, the pinwheel tiling, where $s_{i}$ is an expansion by a linear factor $5^{N_{i} / 2}$.) We cannot, in general, define a simple rule for deflating and rescaling by a fixed factor, and simply iterate this rule. We must be more subtle. However, in the end, everything does work out. 


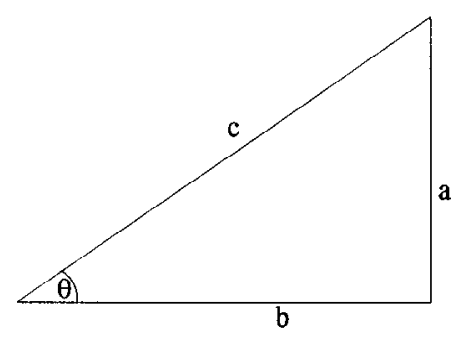

Fig. 2. The basic triangle.

Our basic prototile is a right triangle, with base $b$, altitude $a$, and hypotenuse $c$, as shown in Fig. 2. We refer to the base as the "long" leg, although in principle we might have $b \leq a$. At this point we put no restrictions on the acute angle $\theta=\sin ^{-1}(a / c)$. We divide $T$ into five triangles as in Fig. 3. Subtriangles $1-4$ are similar to $T$, but are smaller by a linear factor of $b / 2 c$. Subtriangle 5 is also similar to $T$, but is smaller than $T$ by a linear factor of $a / c$. This completes the first subdivision of $T$. In the $n$th subdivision of $T$, we take the largest triangles in $T_{n-1}$, and subdivide each of them into five similar triangles.

The pattern of subdivision depends on the angle $\theta$. For example, if $a>b / 2$, then the largest triangle in $T_{1}$ has hypotenuse $a$, and only this triangle is subdivided in step 2 . Thus in $T_{2}$ there are nine triangles, one with hypotenuse $a^{2} / c$, four with hypotenuse $a b / 2 c$, and four with hypotenuse $b / 2$, as shown in Fig. 4 . The third subdivision depends on whether $b / 2>a^{2} / c$, in which case the four triangles of hypotenuse $b / 2$ are subdivided next, or whether $a^{2} / c>b / 2$, in which case the one triangle of hypotenuse $a^{2} / c$ is subdivided once again.

The subdivision of a triangle with $b / 2>a$ is shown in Fig. 5. $T_{2}$ has twenty-one triangles, sixteen of hypotenuse $b^{2} / 4 c$, four of hypotenuse $a b / 2 c$, and one of hypotenuse $a$.

Finally, if $b=2 a$, then all five triangles in $T_{1}$ have the same size. All five are subdivided in the next stage, yielding $T_{2}$ with 25 congruent triangles, all of which are then subdivided to give $T_{3}$ with 125 congruent triangles, and so on. This is the pinwheel tiling of Conway and Radin [R1].

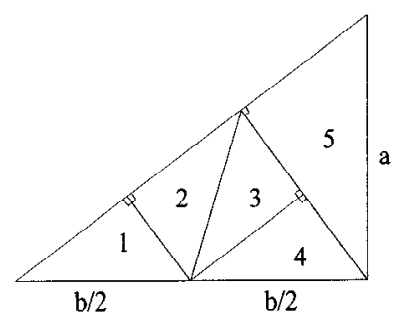

Fig. 3. The substitution rule. 


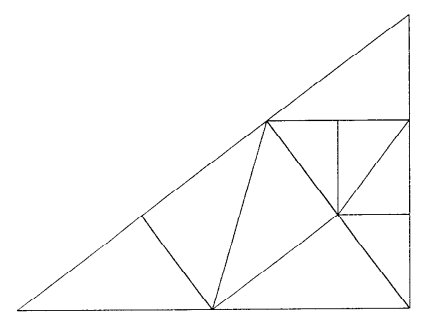

Fig. 4. Two subdivisions of a triangle with $a>b / 2$.

We will show that, for any angle $\theta$, this subdivision scheme generates nonperiodic tilings of the plane. We first need three technical lemmas:

Lemma 1. In $T_{n}$, the ratio of the hypotenuse of the largest tile to the hypotenuse of the smallest tile is strictly less than $\max (c / a, 2 c / b)$.

Proof. The proof is by induction on $n$. It is clearly true for $T_{1}$. Now suppose that in $T_{k}$ the largest tile has hypotenuse $H$, while the smallest tile has hypotenuse $h$, with $H / h<\max (c / a, 2 c / b)$. After subdividing all the triangles with hypotenuse $H$, the largest hypotenuse is strictly less than $H$, while the smallest hypotenuse is the smallest of $h, a H / c$, and $b H / 2 c$. The ratio of largest to smallest hypotenuses in $T_{k+1}$ is therefore strictly less than the largest of $H / h, c / a$, and $2 c / b$.

We often consider an individual tile in $T_{n}$, and examine the effect of further subdivision of $T_{n}$ on that tile. When we write $t \in T_{n}$, we mean that $t$ is a single tile. When we write $t \subset T_{N}$, for some $N>n$, we mean the collection of tiles in $T_{N}$ whose union is $t$. When we write that $t \subset T_{N}$ is similar to $T_{k}$, we mean that the individual tiles in this collection fit together to form the single tile $t \in T_{n}$ in exactly the same pattern that the individual tiles of $T_{k}$ fit together to form $T$.

Lemma 2. Given an integer $n$ and a tile $t \in T_{n}$, there exists an integer $N>n$ such that $t \subset T_{N}$ is the union of more than one tile. That is, every tile in $T_{n}$, no matter how small, is eventually subdivided further.

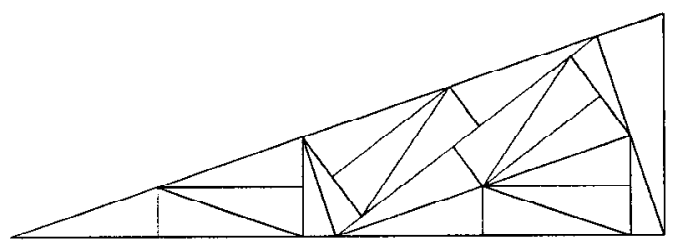

Fig. 5. Two subdivisions of a triangle with $a<b / 2$. 
Proof. The number of tiles grows without bound (increasing by at least four every turn). By Lemma 1 , the area of each tile is at least $\min \left(a^{2} / c^{2}, b^{2} / 4 c^{2}\right)$ times the area of the largest tile. Thus, as $N \rightarrow \infty$, the area of the largest tile in $T_{N}$ must go to zero. For $N$ large enough, this maximal area will be less than the area of $t$, indicating that the tile $t$ has necessarily been subdivided.

Lemma 3. Given an integer $n$, a tile $t \in T_{n}$, and an integer $m$, there exists an integer $N \geq n$ such that $t \subset T_{N}$ is similar to $T_{m}$.

Proof. The proof is by induction on $m$. First we consider $m=1$. Let $N$ be the smallest integer such that $t \in T_{N}$ is subdivided. By Lemma 2, such an $N$ exists. Since $t \subset T_{N-1}$ is a single tile, $t \subset T_{N}$ is the result of taking a single triangle and applying the deflation rule of Fig. 3 once. Thus $t \in T_{N}$ is similar to $T_{1}$.

Now suppose the theorem has been proved for $m=k$, and suppose that $t \subset T_{N_{0}}$ is similar to $T_{k}$. We consider what happens at the $\left(N_{0}+1\right)$ st step. The largest triangles in $T$ get subdivided. This means that either the largest triangles in $t$ get subdivided, or that none of the triangles in $t$ get subdivided. In the first case $t$ will then become similar to $T_{k+1}$, and we are done. In the second case we consider the possibilities for the $\left(N_{0}+2\right)$ nd step, and so on. By Lemma 2, the largest triangle in $t$ must eventually be subdivided, say on the $N$ th turn, so $t \subset T_{N}$ will be similar to $T_{k+1}$.

Definition. A supertile of order $n$ is a collection of tiles that is similar to $T_{n}$. Equivalently, a supertile of order $n$ is a region of the form $e\left(s\left(T_{n}\right)\right)$, where $s$ is a rescaling and $e$ is a Euclidean motion.

Theorem 1. Given any right triangle $T$, there exist tilings of the plane with right triangles similar to $T$, such that any finite set of tiles lies in a supertile, and such that the areas of tiles are bounded both above and below.

Proof. First pick a succession of integers $n_{1}, n_{2}, \ldots$ and tiles $t_{i} \in T_{n_{i}}$. Let $N_{1}=n_{1}$. Pick additional integers $N_{i}, i=2,3, \ldots$, such that, taking $t_{i} \in T_{n_{i}}$ and subdividing $T_{n_{i}}$ an additional $N_{i}-n_{i}$ times, $t_{i} \subset T_{N_{i}}$ is similar to $T_{N_{i-1}}$. By Lemma 3, such integers always exist.

Now pick a triangle similar to $T_{0}$ and place it in the plane. A supertile $S_{1}$ of order $N_{1}$ that contains this triangle in position $t_{1}$ can be constructed. Then a supertile $S_{2}$ of order $N_{2}$ such that $S_{1}$ sits inside $S_{2}$ as $t_{2}$ sits inside $T_{n_{2}}$ is constructed. The process is continued, building supertile $S_{k+1}$ such that $S_{k}$ sits inside $S_{k+1}$ as $t_{k+1}$ sits inside $T_{n_{k+1}}$.

The union of all the supertiles is a tiling of an infinite region. For almost all choices of the $t_{i}$ 's (e.g., having the edges of $t_{i}$ lie in the interior of $T_{n_{i}}$ infinitely often), this region will be the entire plane.

Since the ratio of largest to smallest triangle is uniformly bounded for $S_{n}$ by Lemma 1 , no tile may have a hypotenuse longer than $\max (c / a, 2 c / b)$ times the hypotenuse of a fixed tile, and no tile may have a hypotenuse less than $\min (a / c, b / 2 c)$ times the hypotenuse of the same fixed tile. This provides both an upper and lower bound to the size of the tiles. 
In this construction, many choices were made. Different choices generally lead to different tilings, but these different tilings have many properties in common. Theorems 2 and 3 following apply to all tilings constructed in the manner of Theorem 1.

Theorem 2. In a tiling, the number of orientations in which the basic triangle appears is finite if $\theta / \pi$ is rational and infinite if $\theta / \pi$ is irrational.

Proof. First suppose that $\theta / \pi$ is rational. Consider a tile, positioned as in Fig. 3, with side $b$ along the $x$ axis. Let $P$ denote reflection about the $x$ axis, and let $R_{\alpha}$ denote a counterclockwise rotation by angle $\alpha$. After subdividing once, the orientations of the five daughter tiles, relative to the parent tile, are given by the following elements of $O(2)$ : $R_{\theta} P$ (twice), $R_{\theta}, R_{\pi+\theta}$, and $R_{\pi / 2+\theta} P$. The orientations of tiles in a further subdivision are words in these five elements of $O(2)$. However, with $\theta / \pi$ rational, these five elements generate a finite subgroup of $O(2)$, so only a finite number of orientations can ever appear in a future subdivision.

Since any region of our tiling of the plane sits inside a supertile, any two tiles must have their orientations, relative to the supertile itself, in this group. Thus their orientations, relative to each other, and hence to a fixed reference tile, must lie in the group. Thus only a finite number of orientations can appear in the tiling.

Now suppose that $\theta / \pi$ is irrational. In a basic subdivision, we keep track only of the four triangles of hypotenuse $b / 2$, ignoring the triangle of hypotenuse $a$. When these four triangles divide, we only keep track of the sixteen resultant triangles of side $b^{2} / 4 c$, and so on. In the second generation we find orientations $1=\left(R_{\theta} P\right)^{2}$ and $R_{2 \theta}=R_{\theta}^{2}$, among others. In the $2 n$th generation we find $1, R_{2 \theta}, R_{4 \theta}, \ldots, R_{2 n \theta}$. Since $\theta / \pi$ is irrational, these $2 n+1$ orientations are distinct. Since our tiling of the plane contains supertiles of arbitrarily large size, there is no bound to the number of different orientations that appear.

Theorem 3. In a tiling, the number of sizes in which the basic triangle appears is infinite if $\ln (\sin (\theta)) / \ln [\cos (\theta) / 2]$ is irrational and finite if $\ln (\sin (\theta)) / \ln [\cos (\theta) / 2]$ is rational. In particular, if $\ln (\sin (\theta)) / \ln [\cos (\theta) / 2]=p / q$, with $p$ and $q$ relatively prime integers, then the number of sizes in the tiling is $\max (p, q)$.

Proof. Let $A=a / c$ and let $B=b / 2 c$. If $\ln (A) / \ln (B)$ is irrational, the only way two monomials $A^{a} B^{b}$ and $A^{c} B^{d}$ can be equal is if $a=c$ and $b=d$. We will show that the sizes of triangles in $T_{n}$ (relative to the original triangle) is given by such monomials, and that the number of distinct powers of $A$ grows without bounds as $n \rightarrow \infty$. This will show that the number of distinct sizes grows without bound as $n \rightarrow \infty$.

In each subdivision there are four tiles of size $B$ relative to the parent and one tile of size $A$. Thus the descendants of a given tile all have sizes that are monomials $A^{a} B^{b}$ relative to the ancestor. For every $n>0, T_{n}$ contains at least one tile with size $A^{0} B^{b}$; just take a $B$ child of a $B$ child of $\ldots$ of one of the original $B$ children (or the $B$ child itself, if it has not subdivided). For every $n>0, T_{n}$ contains at least one tile with size $A^{1} B^{b}$; take a $B B B \ldots$ descendant of the original $A$ child. Once $n$ is large enough to have the original $A$ child divide, there is at least one tile with size $A^{2} B^{b}$. In general, once $n$ is 
large enough to allow an $a$ th generation $A A \ldots$ child, it will always have at least one tile whose size has exactly $a$ powers of $A$. This completes the irrational case.

Now suppose that $\ln (A) / \ln (B)=p / q$, with $p$ and $q$ relatively prime. Thus $A^{q}=B^{p}$. Let $r=A^{1 / p}=B^{1 / q}$. Every monomial $A^{a} B^{b}$ is a power of $r$. Assume for the moment that $p \leq q$. By Lemma 1, the ratio of sizes of any two tiles is greater than $B=r^{q}$. Thus only at most $q$ distinct sizes can appear. To see that $q$ sizes $d o$ appear, we note that $A^{0} B^{b_{0}}, A^{1} B^{b_{1}}, \ldots, A^{q-1} B^{b_{q-1}}$ are all distinct powers of $r$.

If $p>q$, Lemma 1 states that the ratio of any two sizes is at least $A=r^{p}$, so at most $p$ different sizes can occur. We produce $p$ different sizes by examining different powers of $B$. In either case, the number of distinct sizes is $\max (p, q)$.

We refer to tilings with $\ln (A) / \ln (B)=p / q$ as $(p / q)$ rational tilings, and denote the class of such tilings as $\operatorname{Til}(p / q)$. For $z$ irrational, we similarly denote the class of tilings with $\ln (A) / \ln (B)=z$ as $\operatorname{Til}(z)$. The different tilings in a class are all derived from the same substitution rule, and have many properties derivable from this rule. When discussing such properties, we sometimes refer to a typical element of the class as "the tiling $\operatorname{Til}(z) .$,

Note that $\ln (\sin (\theta)) / \ln [\cos (\theta) / 2]$ is a strictly decreasing function of $\theta$ on the interval $(0, \pi / 2)$. From this monotonicity, and from the countability of the rationals, it is clear that only a countable set of angles $\theta$ give rise to a finite number of rotations, and only a countable set of angles $\theta$ give rise to a finite number of sizes. The intersection of these two countable sets turns out to be a single point.

Theorem 4. The only angle that gives rise to both a finite number of orientations and a finite number of sizes is $\theta=\pi / 4$. That is, the tiling Til(1/3).

Proof. Let $x=\exp (i \theta)$, with $0<\theta<\pi / 2$. We are looking for solutions to the equation $\sin (\theta)^{q}=[\cos (\theta) / 2]^{p}$, which we rewrite as

$$
2^{q}(x+\bar{x})^{p}=2^{2 p}(-i)^{q}(x-\bar{x})^{q},
$$

where $\bar{x}=\exp (-i \theta)=x^{-1}$. Note that, for fixed $p, q$, there is at most one solution to (2.1) in the first quadrant, $\operatorname{since} \ln [\sin (\theta)] / \ln [\cos (\theta) / 2]$ is monotonic.

Since by assumption $\theta$ is a rational multiple of $\pi, x$ is a primitive $n$th root of unity for some integer $n$. If $x$ is a solution and $q$ is even, then (2.1) has (real) integer coefficients, and all the primitive $n$th roots of unity are also solutions. If $q$ is odd, all the primitive $n$th roots of unity are solutions either to (2.1) or to the conjugate equation

$$
2^{q}(x+\bar{x})^{p}=2^{2 p} i^{q}(x-\bar{x})^{q} .
$$

Equation (2.2), with $q$ odd, has no solutions in the first quadrant, as the right-hand side is positive but the left-hand side is negative. Since (2.1) admits only one solution in the first quadrant, there must be exactly one primitive $n$th root of unity in the first quadrant.

This means that $n$ must equal $5,6,7,8,10,12$, or 18 . Checking these individually, we see that only $n=8$, or $\theta=\pi / 4$, yields a rational value of $\ln [\sin (\theta)] / \ln [\cos (\theta) / 2]$. In that one case $\sin (\theta)=\cos (\theta)=\sqrt{2} / 2$, and $\sin ^{3}(\theta)=\cos (\theta) / 2$. 
In a periodic tiling, all of the sizes and orientations are exhibited in a compact region, so neither the number of sizes nor the number of orientations can be infinite. Thus we have

Corollary. If $z \neq 1 / 3$, then the tiling Til(z) is not periodic.

In fact, it turns out that $\operatorname{Til}(1 / 3)$ is not periodic, either. This is shown in Section 6.

\section{An Example with Two Sizes}

In this section we consider in detail the tiling Til(1/2), shown in Fig. 1. This example is chosen not as a special case, but rather as a simple example of some general phenomena. Based on the statistical analysis of Section 4, we expect all tilings Til $(p / q)$, with $q>1$ and $p / q \neq 1 / 3$, to be qualitatively similar to Til(1/2). Specifically, in all these cases the population matrix has two or more eigenvalues with modulus bigger than one. This causes fluctuations in the statistical composition of supertiles $S_{n}$ to grow with $n$. This, in turn, can cause the tiling to fail to be globally edge-to-edge.

Theorem 5. Til(1/2) is a tiling with two sizes of tiles, each of which appears in an infinite number of orientations. The substitution scheme has the sibling edge-to-edge property, but the tiling is not globally edge-to-edge. Specifically, the tiles meet in an infinite number of ways.

Proof. The existence of the tiling, the number of sizes, and the number of orientations follow from Theorems 1-3. The sibling edge-to-edge property is manifest, if we consider the basic tile to have four vertices - the three obvious ones and the midpoint of the long leg. The difficulty is in proving that tiles meet in an infinite number of distinct ways.

The process by which this happens is as follows. There are certain special lines in the tiling. The long diagonal in Fig. 1 is an example. On each side of such a line there are triangles, all of the same size, whose hypotenuses or long legs make up part of the long line. The pattern is different on the two sides of the line, with one side having (say) more legs in a certain region and the other side having more hypotenuses. This imbalance causes the tiles on one side of the line to appear shifted relative to those on the other side, a phenomenon we call "slippage." We will exhibit regions where the imbalance is arbitrarily large. Since the imbalance is unbounded, the slippage of one side relative to the other reaches arbitrarily high multiples of $b$, modulo $c$, where $b$ and $c$ are the lengths of the long leg and hypotenuse. Since $b / c$ is irrational, this gives rise to an infinite number of ways in which one triangle can meet another across the long line.

Lines where slippage occurs are called "fault lines." Note that each fault line has only finite length, and allows only a finite amount of slippage. However, we will find fault lines of arbitrarily long length with arbitrarily much slippage. This precludes there being a finite bound on how many ways one triangle can meet another.

The Til(1/2) tiling is based on a right triangle with legs $a=\sqrt{2(\sqrt{17}-1)}$ and $b=\sqrt{17}-1$ and hypotenuse $c=4$. Several degrees of subdivision are shown in Fig. 6 . 


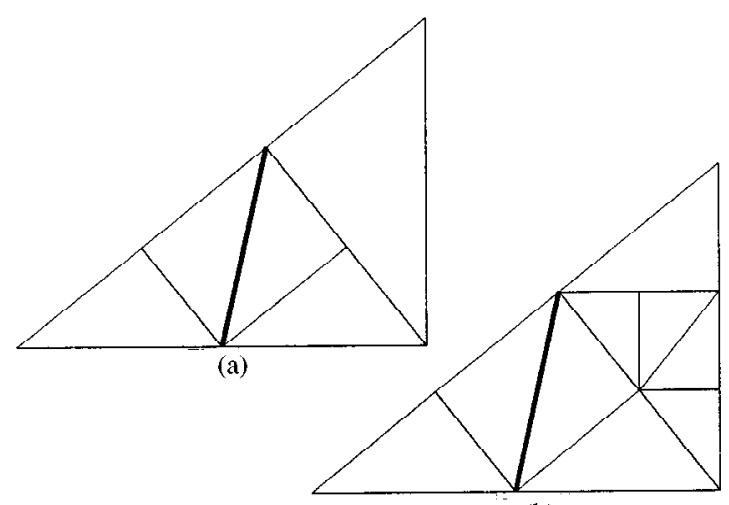

(b)

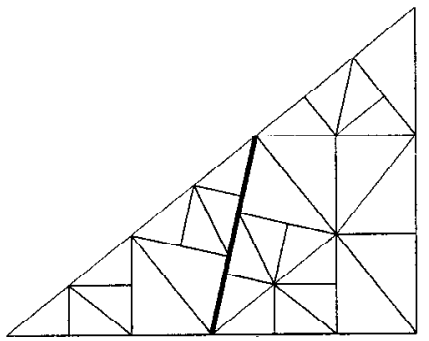

(c)

Fig. 6. Three stages of subdivision for $\operatorname{Til}(1 / 2)$.

At each level there are two sizes of triangle, whose linear sizes differ by a factor of $a / c$. The heavily shaded lines in Fig. 6 are fault lines. For each $T_{n}$, with $n$ even, only large triangles abut the illustrated fault line. Once a fault line is formed, the triangles on opposite sides of the line evolve separately, and begin to slip. We shall prove that this slippage increases without bound.

Til (1/2) may be viewed as a traditional substitution system, with two prototiles, which we call $B$ and $S$ (for big and small). Each subdivision, followed by rescaling by $c / a$, may be viewed as a replacement of each $S$ triangle by a $B$ triangle, and replacement of each $B$ triangle by a $B$ triangle and four $S$ triangles. Let the population of $T_{n}$ be

$$
\Psi_{n}=\left(\begin{array}{c}
N_{B} \\
N_{S}
\end{array}\right),
$$

where $N_{B}$ and $N_{S}$ are the numbers of big and small tiles. $\Psi$ satisfies $\Psi_{n+1}=M \Psi_{n}$, where the population matrix is

$$
M=\left(\begin{array}{ll}
1 & 1 \\
4 & 0
\end{array}\right),
$$

with eigenvalues $\lambda_{ \pm}=(1 \pm \sqrt{17}) / 2$ and eigenvectors

$$
\zeta_{ \pm}=\left(\begin{array}{c}
\sqrt{17} \pm 1 \\
\pm 8
\end{array}\right)
$$


As $n$ grows, the ratio of $N_{B}$ to $N_{S}$ approaches $(\sqrt{17}+1) / 8 \approx 0.6404$. The exact populations are

$$
\begin{aligned}
N_{B}(n) & =\frac{1}{\sqrt{17}}\left(\left(\frac{\sqrt{17}+1}{2}\right)^{n+1}-\left(\frac{1-\sqrt{17}}{2}\right)^{n+1}\right), \\
N_{S}(n)=4 N_{B}(n-1) & =\frac{4}{\sqrt{17}}\left(\left(\frac{\sqrt{17}+1}{2}\right)^{n}-\left(\frac{1-\sqrt{17}}{2}\right)^{n}\right) .
\end{aligned}
$$

Note that $\left|\lambda_{-}\right|>1$, so that $\left|8 N_{B}-(\sqrt{17}+1) N_{S}\right|$ grows with $n$.

Next we consider what happens along a fault line. To do this we must consider the boundary of $T_{2 n}$. Note that the hypotenuse and long leg of $T_{2}$ consist only of hypotenuses and long legs of big triangles. Applying the subdivision again, we get that the hypotenuse and long leg of $T_{4}$ also consists only of hypotenuses and long legs of big triangles. Similarly for all $T_{2 n}$.

The evolution of these legs and hypotenuses is a substitution system in its own right, only in one dimension. There are four symbols, $H^{+}, H^{-}, L^{+}$, and $L^{-}$, representing the two orientations of the hypotenuse and long leg, respectively. From Fig. 6(b), we see that the substitution rule, which we denote $\sigma_{0}$, is

$$
\sigma_{0}\left(H^{+}\right)=L^{+} L^{-} H^{+}, \quad \sigma_{0}\left(H^{-}\right)=H^{-} L^{+} L^{-}, \quad \sigma_{0}\left(L^{ \pm}\right)=H^{ \pm} H^{ \pm} .
$$

Since $L^{+}$and $L^{-}$only appear in the combination $L^{+} L^{-}$, we can define a new symbol $L=L^{+} L^{-}$and have a substitution system with three elements, whose rule we denote $\sigma:$

$$
\sigma\left(H^{+}\right)=L H^{+}, \quad \sigma\left(H^{-}\right)=H^{-} L, \quad \sigma(L)=H^{+} H^{+} H^{-} H^{-} .
$$

Lemma 4. The sequence $\sigma^{n}\left(H^{+}\right)$contains neither the subsequence LL nor the subsequence $H^{-} H^{+}$.

Proof. The proof is simple induction. The only way to generate an $L L$ is from an $H^{-} H^{+}$, and the only way to generate an $H^{-} H^{+}$is from $L L$. Since neither appear in the first generation, neither appears in any subsequent generation.

Lemma 5. The sequence $\sigma^{n}\left(H^{+}\right)$does not contain a subsequence of more than six consecutive H's.

Proof. Since $L L$ does not occur in $\sigma^{n-1}\left(H^{+}\right)$, the longest possible sequence of $H$ 's in $\sigma^{n}\left(H^{+}\right)$would come from a sequence $H^{+} L H^{-}$in $\sigma^{n-1}\left(H^{+}\right)$. This gives rise to $L H^{+} H^{+} H^{+} H^{-} H^{-} H^{-} L$, or six $H$ 's in a row.

Let $f(n)$ equal the number of $L$ 's in the first half of the sequence $\sigma^{n}\left(H^{+}\right)$minus the number of $L$ 's in the second half of the sequence. As we shall see, $f(n)$ is closely related to the extent to which slippage occurs along the largest fault line in $T_{2 n+2}$. 
Lemma 6. If $|f(n)|>6$, then $|f(n+1)| \geq|f(n)|+2$.

Proof. Let $s$ and $s^{\prime}$ denote the first and second halves of $\sigma^{n}\left(H^{+}\right)$, respectively. Suppose that $s$ contains $h H$ 's and $l L$ 's, while $s^{\prime}$ contains $h^{\prime} H$ 's and $l^{\prime} L$ 's. Note that $f(n)=$ $l-l^{\prime}=h^{\prime}-h$. Since each $H$ generates an $H$ and an $L$, while each $L$ generates four $H$ 's, $\sigma(s)$ contains $4 l+h H$ 's and $h L$ 's, while $\sigma\left(s^{\prime}\right)$ contains $4 l^{\prime}+h^{\prime} H$ 's and $h^{\prime} L$ 's. Thus $\sigma(s)$ contains $2 f(n)$ more terms, but $f(n)$ fewer $L$ 's, than $\sigma\left(s^{\prime}\right)$.

Now suppose $f(n)>0$. The first half of $\sigma^{n+1}\left(H^{+}\right)$is all of $\sigma(s)$, minus the last $f(n)$ elements, while the second half of $\sigma^{n+1}\left(H^{+}\right)$is the last $f(n)$ elements of $\sigma(s)$ and all of $\sigma\left(s^{\prime}\right)$. Thus $f(n+1)$ equals $-f(n)$ minus twice the number of $L$ 's in the last $f(n)$ elements of $\sigma(s)$. Since $f(n)>6$, there must be at least one $L$ in the last $f(n)$ elements of $\sigma(s)$, so $|f(n+1)|=-f(n+1) \geq f(n)+2$.

If $f(n)<0$, then the first half of $\sigma^{n+1}\left(H^{+}\right)$is all of $\sigma(s)$, plus the first $|f(n)|$ elements of $\sigma\left(s^{\prime}\right)$. We then have $f(n+1)$ equalling $-f(n)$ plus twice the number of $L$ 's in the first $|f(n)|$ elements of $\sigma(s)$. By Lemma 5, there must be at least one such $L$, so $|f(n+1)|=f(n+1) \geq 2-f(n)=|f(n)|+2$.

Lemma 7. $\lim _{n \rightarrow \infty}|f(n)|=+\infty$.

Proof. By explicit computation, $f(1)=1, f(2)=-1, f(3)=1, f(4)=-3$, $f(5)=3, f(6)=-5$, and $f(7)=9$. By Lemma 6 , for $n \geq 7,|f(n+1)|>|f(n)|$, so $|f(n)| \geq n+2$ goes to infinity as $n \rightarrow \infty$.

We have proven that $|f(n)|$ grows without bound, which is all that we need. In fact, $|f(n)|$ grows exponentially. For large $n$, an approximate fraction $2 /(3+\sqrt{17})$ of the elements of $\sigma^{n}\left(H^{+}\right)$are $L$ 's, so $f(n+1) \approx-f(n)+2 f(n) \times 2 /(3+\sqrt{17})=$ $f(n)(1-\sqrt{17}) / 2$. The growth rate, $(1-\sqrt{17}) / 2$, equals $\lambda_{-}$, the second eigenvalue of the population matrix $M$.

We now return to the question of slippage along fault lines. Consider two large triangles that meet hypotenuse to hypotenuse to form a rectangle, as in Fig. 7. Let $P$ and $R$ be the ends of the common hypotenuse, and let $Q$ be the midpoint. Rotation by $\pi$ about $Q$ sends each triangle into the other.

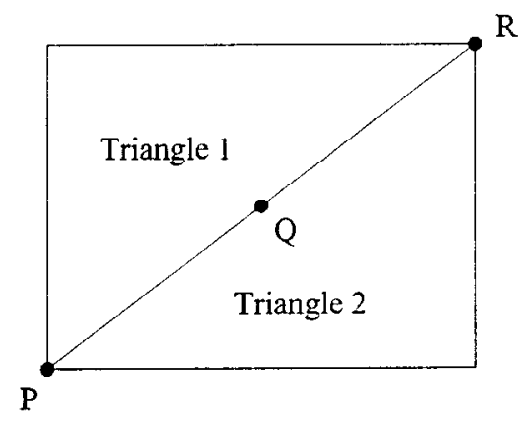

Fig. 7. A fault line. 
Subdivide the pair of triangles $2 n$ times. The pattern of subdivision of triangle 2 along the interval $P Q$ is the same as that of triangle 1 along the interval $R Q$. We have already seen that only large triangles abut the main diagonal, and that they do so only along their hypotenuses and long legs. For each point $x$ on the interval $P Q$, let $g_{n}(x)$ be the number of complete long legs on the triangle 1 side of $P x$ minus the number of complete long legs on the triangle 2 side of $P x$.

Lemma 8. $\left|g_{n}(Q)\right| \geq(c / b)|f(n)|-1$. In particular, $\lim _{n \rightarrow \infty}\left|g_{n}(Q)\right|=\infty$.

Proof. Consider the $2 n$-fold subdivision of the pair of triangles in Fig. 7. The hypotenuse of triangle 2 gets divided into $\sigma^{n}\left(H^{+}\right)$. Let $M_{n}$ be the point on the hypotenuse corresponding to the middle of the sequence $\sigma^{n}\left(H^{+}\right)$. By construction, there are $2 f(n)$ more long legs, and $f(n)$ fewer hypotenuses, between $P$ and $M_{n}$ than between $M_{n}$ and $R$. Since legs have length $b$, hypotenuses have length $c$, and $2 b>c, M_{n}$ lies a distance $f(n)(2 b-c)$ closer to $R$ than to $P$, or a distance $f(n)(2 b-c) / 2$ beyond $Q$.

Suppose $f(n)<0$. Then $M_{n}$ lies between $P$ and $Q$. The number of hypotenuses between $P$ and $Q$ is at least the number of hypotenuses between $P$ and $M_{n}$, and so is at least $-f(n)$ more than the number of hypotenuses between $Q$ and $R$. Since the length of $P Q$ equals that of $Q R$, and length equals $b \times \operatorname{legs}$ plus $c \times$ hypotenuses, there are at least $-(c / b) f(n)$ more legs in $Q R$ than in $P Q$. Thus $\left|g_{n}(Q)\right|$ is at least the integer part of $(c / b)|f(n)|$, which is greater than $(c / b) f(n)-1$.

If $f(n)>0$ this situation is reversed. Then $M_{n}$ lies between $Q$ and $R$, and the interval between $M_{n}$ and $R$ has the surplus of $f(n)$ hypotenuses. The interval $Q R$ has a surplus of at least that many hypotenuses, so the interval $P Q$ has a surplus of at least $(c / b) f(n)$ legs. Taking the integer part, we see that $P Q$ has a surplus of at least $(c / b) f(n)-1$ complete legs.

We are now in a position to prove Theorem 5. Let $x$ be a point on $P Q$ that is the vertex of a tile in the subdivision of triangle 2 , and let $y$ be the nearest vertex, on $P R$, of a tile in the subdivision of triangle 1. The interval $x y$ is the interval of contact between two tiles, one in triangle 1 and the other in triangle 2. If $y$ lies between $P$ and $x$, then the length of $x y$ is congruent, modulo $c$, to $b g_{n}(x)$. If $x$ lies between $P$ and $y$, then the length is congruent either to $-b g_{n}(x)$ or $b-b g_{n}(x)(\bmod c)$, depending on whether $x y$ is part of the hypotenuse or long leg of a tile on side 1 .

As we pick points $x$ from $P$ to $Q, g_{n}(x)$ goes, by steps of one, from 0 to $g_{n}(Q)$. Thus the intervals $x y$ take on at least $\left|g_{n}(Q)\right| / 2$ distinct lengths. Since $\left|g_{n}(Q)\right|$ grows with $n$, all we need for Theorem 5 is to show that fault lines modeled on $\sigma^{n}\left(H^{+}\right)$, for arbitrarily large values of $n$, occur in the tiling $\operatorname{Til}(1 / 2)$.

By construction, $\operatorname{Til}(1 / 2)$ contains supertiles modeled on $T_{m}$, for arbitrarily large values of $m$. If $m$ is even, then the primary fault line exhibited in Fig. 6 is modeled on $\sigma^{n}\left(H^{+}\right)$, with $m=2 n+2$. If $m$ is odd, then there is also a supertile modeled on $T_{m-1}$, namely the descendants of the large tile in the first subdivision of $T$. Since $m-1$ is even, this supertile contains a fault line modeled on $\sigma^{n}\left(H^{+}\right)$, with $m=2 n+3$. Since $m$ is unbounded, we have obtained our requisite arbitrarily long fault lines.

This completes the proof of Theorem 5 . 


\section{Statistical Properties of Rational Tilings}

In this section we consider the statistical distribution of sizes and orientations of tiles in the various rational tilings $\operatorname{Til}(p / q)$. More precisely, we consider the distribution of sizes and orientations in a supertile modeled on $T_{n}$, and take the limit as $n \rightarrow \infty$. We first show that the distribution of sizes approaches a simple limit as $n \rightarrow \infty$. The limiting distribution is given by the eigenvector associated to the largest eigenvalue of a $p \times p$ (or $q \times q$ ) population matrix. We also analyze the second eigenvalue of this matrix. The failure of the edge-to-edge property for $\operatorname{Til}(1 / 2)$ was a result of fluctuations that were governed by this second eigenvalue. We conjecture that the edge-to-edge property holds only for those tilings with second eigenvalue smaller than one, and we classify these tilings.

We then turn to the joint distribution of sizes and orientations in rational $p / q$ tilings. We show that, for each size, the distribution of orientations is asymptotically uniform. Specifically, we parametrize $O(2)$, the group of orientations, by two copies of the unit circle. Given an interval in this set, the fraction of tiles in $T_{n}$, of a given size, whose orientations lie in that interval, approaches a constant times the length of the interval. In the terminology of Radin [R3], the tilings $T(p / q)$ exhibit "statistical rotational symmetry."

By Theorem 3, $\operatorname{Til}(p / q)$ contains triangles of $m=\max (p, q)$ distinct sizes. Til $(p / q)$ is equivalent to a traditional substitution tiling, with prototiles $D_{1}, \ldots, D_{m}$ of $m$ sizes. We take $D_{1}$ to be the largest size and $D_{m}$ to be the smallest. Subdivision and linear rescaling by $r^{-1}$, where $r=(a / c)^{1 / p}=(b / 2 c)^{1 / q}$, takes $D_{i+1}$ to $D_{i}$, and takes $D_{1}$ to four copies of $D_{q}$ and one copy of $D_{p}$. That is, the population matrix, which gives the population of $T_{n+1}$ in terms of the population of $T_{n}$, has matrix elements

$$
M_{i j}= \begin{cases}1 & \text { if } j=i+1 \\ 1 & \text { if } j=1 \text { and } i=p \\ 4 & \text { if } j=1 \text { and } i=q \\ 0 & \text { otherwise. }\end{cases}
$$

The properties of $M$ are summarized in the following theorem:

\section{Theorem 6.}

(1) The characteristic polynomial of $M$ is

$$
p(\lambda)=\left\{\begin{array}{lll}
\lambda^{q}-\lambda^{q-p}-4 & \text { if } & p<q \\
\lambda^{p}-4 \lambda^{p-q}-1 & \text { if } & p>q .
\end{array}\right.
$$

(2) The largest eigenvalue of $M$ is $r^{-2}$.

(3) There are exactly $q$ eigenvalues with modulus greater than one.

(4) The eigenvectors $\psi$ of $M$, for fixed eigenvalue $\lambda$, take the form

$$
\psi_{k}=\lambda^{k}-\lambda^{k-p} H(k-p-1)-4 \lambda^{k-q} H(k-q-1),
$$

where $H(n)$ is the discrete Heaviside function

$$
H(n)= \begin{cases}1 & \text { if } \quad n \geq 0 \\ 0 & \text { otherwise }\end{cases}
$$


(5) Asymptotically, the number of tiles of size $D_{k}$ is a fraction

$$
v_{k}=\frac{1-r^{2}}{4 c^{2}}\left(a^{2} H(p-k)+b^{2} H(q-k)\right) r^{-2 k}
$$

of the total.

(6) Asymptotically, the area covered by tiles of size $D_{k}$ is a fraction

$$
\rho_{k}=\frac{a^{2} H(p-k)+b^{2} H(q-k)}{p a^{2}+q b^{2}}
$$

of the total.

Note that if $p<q$, then $H(k-q-1)$ is identically zero and $H(q-k)$ is identically one. If $p>q$, then $H(k-p-1)$ is identically zero and $H(p-k)$ is identically one. As written, expressions (4.3), (4.5), and (4.6) apply to both the $p<q$ and $p>q$ cases.

Corollary. Let $t$ be a tile of size $k$. Let $N_{k}(n)$ be the number of descendants of $t$ after the $n$th application of the substitution rule. Then

$$
\lim _{n \rightarrow \infty} r^{2 n} N_{k}(n)=\frac{4 c^{2} r^{2 k}}{\left(1-r^{2}\right)\left(p a^{2}+q b^{2}\right)} .
$$

Proof of the Corollary. From the distribution (4.5), we compute the average area per tile to be $\left(1-r^{2}\right) a b\left(p a^{2}+q b^{2}\right) / 8 c^{2}$. A tile of size $D_{k}$, subdivided and rescaled $n$ times, has area $a b r^{2 k-2 n} / 2$. Dividing by the area per unit tile we obtain (4.7).

Remark. If $q>1$, then the second largest eigenvalue of $M$ is greater than one. The fluctuations in population associated to the corresponding eigenvector then grow with subdivision, although they do not grow as fast as the population as a whole. It was precisely this phenomenon that caused the edge-to-edge property to fail for Til( $1 / 2)$. If $q=1$, then the second eigenvalue is less than one. In Section 6 we shall see that, in $\operatorname{Til}(2)$, this causes the triangles to meet in only a finite number of ways.

Conjecture. If $p$ and $q$ are relatively prime integers, $q>1$ and $p / q \neq 1 / 3$, then the tiling Til $(p / q)$ has tiles that meet in an infinite number of different ways. If $p>1$, then the tiles in Til $(p)$ meet in only a finite number of ways.

Proof of Theorem 6. For $k<m$, the $k$ th row of the vector equation $M \psi=\lambda \psi$ reads

$$
\psi_{k+1}=\lambda \psi_{k}-\left(\delta_{k, p}+4 \delta_{k, q}\right) \psi_{1} .
$$

Setting $\psi_{1}=\lambda$, we repeatedly use (4.8) to obtain expression (4.3) for $\psi_{2}, \ldots, \psi_{m}$. Plugging this into the $m$ th row of $M \psi=\lambda \psi$ then gives the characteristic polynomial (4.2).

Now suppose $p<q$, and consider the function $p(\lambda)$ for $\lambda$ real and positive. Note that $p\left(r^{-2}\right)=r^{-2 q}-r^{2 p-2 q}-4=4 c^{2} / b^{2}-4 a^{2} / b^{2}-4=0$, since $a^{2}+b^{2}=c^{2}$. When $\lambda \geq 1, p^{\prime}(\lambda)=q \lambda^{q-1}-(q-p) \lambda^{q-p-1}>0$. Thus $p(\lambda)>0$ for all $\lambda>r^{-2}$. 
Now let $\lambda \neq r^{-2}$ be a root of $p(\lambda)$. We show that $|\lambda|<r^{-2}$. If $\lambda$ is real and positive, then $\lambda<r^{-2}$. If $\lambda$ is not real and positive, then, since $q$ and $q-p$ are relatively prime, we cannot have $\lambda^{q}$ and $\lambda^{q-p}$ both real and positive. Thus, by the triangle inequality,

$$
0=|p(\lambda)|=\left|\lambda^{q}-\lambda^{q-p}-4\right|>\left|\lambda^{q}\right|-\left|\lambda^{q-p}\right|-4=p(|\lambda|) .
$$

Since $p(|\lambda|)<0$, we must have $|\lambda|<r^{-2}$.

We count the number of roots in the unit circle via the argument principle, tracking the argument of $p(\exp (i \theta))$ as $\theta$ goes from 0 to $2 \pi$. When $|\lambda|=1, p(\lambda)$ always has negative real part, as $4>\left|\lambda^{q}+\lambda^{q-p}\right|$. Thus the winding is zero, and none of the roots of $p(\lambda)$ lie in the unit circle. Thus there are $q$ roots, counted with multiplicity, outside the unit circle. Since $p^{\prime}(\lambda)$ is never zero outside the unit circle, all these roots are distinct.

Now suppose $p>q$. Then $p(\lambda)=\lambda^{p-q}\left(\lambda^{q}-\lambda^{q-p}-4\right)$. By the same arguments as before, $p\left(r^{-2}\right)=0$, and $p(\lambda)>0$ for real $\lambda>r^{-2}$. For any eigenvalue $\lambda$ other than $r^{-2}$, $0=|p(\lambda)|>p(|\lambda|)$. Since $p(|\lambda|)<0,|\lambda|<r^{-2}$. By the argument principle, there are $p-q$ roots inside the unit circle, since the dominant term of $p(\lambda)$ on the unit circle is $4 \lambda^{p-q}$. This leaves $q$ roots outside the unit circle. This completes the proof of statements (1)-(4).

To obtain the asymptotic distribution of sizes, we must decompose the initial population into eigenvectors of $M$. The asymptotic distribution will be the eigenvector corresponding to the largest eigenvalue, assuming the coefficient of that eigenvector is nonzero. We have shown that this largest eigenvalue is $r^{-2}$. Since the total area of the system grows by a factor of $r^{-2}$ each time, the coefficient of this eigenvector is not zero. Thus the asymptotic distribution of population is given by a multiple of the expression (4.3), with $\lambda=r^{-2}$. Normalizing, we obtain expression (4.6). Multiplying this by the area of each tile and normalizing again gives the asymptotic distribution of areas (4.7).

We now turn to the joint distribution of sizes and orientation. To do this we must first parametrize the space of possible orientations of a single size. This space is isomorphic to two copies of the unit circle. We specify both the handedness of the triangle and the direction a fixed vector in it points in the plane. We take as our reference vector the ray from the small angle to the right angle. In Fig. 8, the first triangle has orientation $\left(+, \varphi_{1}\right)$, while the second has orientation $\left(-, \varphi_{2}\right)$. We let $\Omega$ denote the ordered pair $( \pm, \varphi)$, and let $d \Omega=d \varphi / 4 \pi$ be the Haar measure on the space of orientations. The space of all possible tiles up to translation, which we denote $X$, is $2 m$ copies of $S^{1}$.

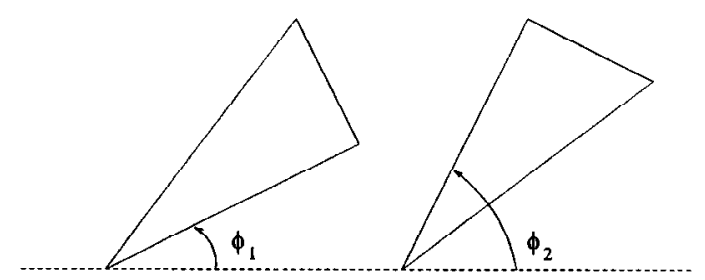

Fig. 8. Orientation of triangles. 
Let $f$ be a function on $X$. Given a collection $S$ of tiles, let $\langle f, S\rangle$ be the average value of $f$ on the individual tiles in $S$, where each tile is given equal weight. Let $\langle f, S\rangle^{\prime}$ be the average value of $f$ on the individual tiles in $S$, where each tile is weighted proportionally to its area.

Theorem 7. Assume a tiling $\operatorname{Til}(p / q)$, with $p$ and $q$ relatively prime and $p / q \neq 1 / 3$. Let $f$ be a continuous function on $X$, and let $\left\{S_{n}\right\}$ be a sequence of supertiles of increasing size. Let $d v=\sum_{k} v_{k} d \Omega_{k}$ and $d \rho=\sum_{k} \rho_{k} d \Omega_{k}$ be measures on $X$, where $v_{k}$ and $\rho_{k}$ are as in Theorem 6. Then

$$
\begin{aligned}
& \lim _{n \rightarrow \infty}\left\langle f, S_{n}\right\rangle=\int_{X} f d \nu, \\
& \lim _{n \rightarrow \infty}\left\langle f, S_{n}\right\rangle^{\prime}=\int_{X} f d \rho .
\end{aligned}
$$

These limits also apply if $f$ is the characteristic function of an interval in $X$.

Proof. We first reduce the problem to establishing (4.10a) for an arbitrary continuous function $f$. Once we have established (4.10a), (4.10b) follows by applying (4.10a) to the continuous function $\tilde{f}(k, \Omega)=r^{2 k} f(k, \Omega)$. Once (4.10a,b) have been established for continuous functions, the extension to characteristic functions is standard (for details see [CFS]). Let $I$ be an interval, and let $\chi_{I}$ be its characteristic function. We choose continuous functions $f_{\varepsilon}^{ \pm}$, such that $f_{\varepsilon}^{-} \leq \chi_{I} \leq f_{\varepsilon}^{+}$, and such that $\lim _{\varepsilon \rightarrow 0} \int_{X} f_{\varepsilon}^{ \pm} d v=$ $\int_{I} d \nu \cdot \lim _{n \rightarrow \infty}\left\langle\chi_{I}, S_{n}\right\rangle$ is sandwiched between $\lim _{n \rightarrow \infty}\left\langle f_{\varepsilon}^{-}, S_{n}\right\rangle$ and $\lim _{n \rightarrow \infty}\left\langle f_{\varepsilon}^{+}, S_{n}\right\rangle$, hence between $\int_{X} f_{\varepsilon}^{-} d \nu$ and $\int_{X} f_{\varepsilon}^{+} d \nu$, and so must equal $\int_{I} d \nu$.

To establish (4.10a) we must introduce some notation. If $f$ is a function on $X$ and $S$ is a collection of tiles, let $(f, S)$ be the sum of $f$ evaluated on the individual tiles of $S$. Let $\Phi$ denote the action of subdividing and rescaling. That is, $\Phi$ acting on a tile of size $D_{k+1}$ gives a tile of size $D_{k}$ of the same orientation, while $\Phi$ acting on a tile of size $D_{1}$ gives one tile of size $D_{p}$ and four of size $D_{q}$, having various orientations. Let $\Phi^{*}$ be the dual of $\Phi$ by $(\cdot, \cdot)$, acting on the space of functions:

$$
\left(\Phi^{*} f, S\right)=(f, \Phi(S)) .
$$

Let $f_{n}=\left(\Phi^{*}\right)^{n} f$. Note that $\Phi^{*}$ is linear and sends nonnegative functions to nonnegative functions, so if $f \leq g$, then $f_{n} \leq g_{n}$.

Now suppose we have a sequence of tiles $t_{n}$ and supertiles $S_{n}=\Phi^{n} t_{n}$. We have that

$$
\left\langle f, S_{n}\right\rangle=\frac{\left(f, S_{n}\right)}{\# \text { of tiles in } S_{n}}=\frac{\left(f_{n}, t_{n}\right)}{\# \text { of tiles in } S_{n}} .
$$

Since the number of tiles is given asymptotically by (4.7), (4.10a) is equivalent to $r^{2 n} f_{n}$ converging uniformly to $\int_{X} f d v$ times

$$
\zeta_{0}(k, \Omega)=\frac{4 c^{2} r^{2 k}}{\left(1-r^{2}\right)\left(p a^{2}+q b^{2}\right)} .
$$

We examine the spectrum of the linear operator $\Phi^{*}$ on the function space $C(X)$. The key lemma, whose proof we defer, follows. 
Lemma 9. The spectrum of $\Phi^{*}$ is pure point. $\zeta_{0}$ is an eigenfunction with eigenvalue $r^{-2}$. All other eigenvalues have norm strictly less than $r^{-2}$. Any continuous function $f$ can be written as a (possibly infinite) sum of eigenfunctions of $\left(\Phi^{*}\right)^{|q-p|}$, such that a subsequence of partial sums converges uniformly to $f$.

Remark. If $p<q$, it turns out that there are a number of functions $\zeta$ for which $\left(\Phi^{*}\right)^{|q-p|} \zeta=0$, but $\Phi^{*} \zeta \neq 0$. Thus, to achieve a basis for the space of continuous functions, we must use eigenfunctions of $\left(\Phi^{*}\right)^{|q-p|}$ rather than just eigenfunctions of $\Phi^{*}$.

Given the lemma, we write

$$
f=\sum_{i=0}^{\infty} c_{i} \zeta_{i}
$$

Since a subsequence of the partial sums converges uniformly, and since $\zeta_{0}$ has a positive minimum, for each $\varepsilon>0$ we can find an integer $N$ such that

$$
f^{-} \equiv\left(\sum_{i=0}^{N} c_{k} \zeta_{i}\right)-\varepsilon \zeta_{0}<f<\left(\sum_{i=0}^{N} c_{k} \zeta_{i}\right)+\varepsilon \zeta_{0} \equiv f^{+}
$$

where each $\zeta_{i}$ is an eigenfunction with eigenvalue $\lambda_{i}$. (Strictly speaking, $\zeta_{i}$ is merely an eigenfunction of $\left(\Phi^{*}\right)^{|q-p|}$, not necessarily of $\Phi^{*}$, but this distinction makes no difference.) Since $\left|r^{-2} \lambda_{i}\right|<1$ for all $i>0$, for $n>|p-q|$ we have

$$
\left(c_{0}-\varepsilon\right) \zeta_{0}+\sum_{i=1}^{N} c_{k}\left(r^{2} \lambda_{i}\right)^{n} \zeta_{i} \leq r^{2 n} f_{n} \leq\left(c_{0}+\varepsilon\right) \zeta_{0}+\sum_{i=1}^{N} c_{k}\left(r^{2} \lambda_{i}\right)^{n} \zeta_{i}
$$

As $n \rightarrow \infty$, the left-hand side converges uniformly to $\left(c_{0}-\varepsilon\right) \zeta_{0}$, while the right-hand side converges uniformly to $\left(c_{0}+\varepsilon\right) \zeta_{0}$. Since $\varepsilon$ is arbitrary, $r^{2 n} f_{n}$ must converge uniformly to $c_{0} \zeta_{0}$.

All that remains is to compute $c_{0}$ in terms of $f$. Since $\zeta_{0}$ is invariant under rotation and reflection, $c_{0}$ must be of the form $\sum_{k} d_{k} \int f(k, \Omega) d \Omega$ for some universal constants $d_{k}$. By comparing characteristic functions of different sizes, we see that the constants $d_{k}$ must be proportional to $v_{k}$. Finally, for the constant function $f=1, f_{n}(k, \Omega)$ is the number of descendants of a tile of size $D_{k}$, which we have already computed in (4.7). This fixes the proportionality constant.

Proof of Lemma 9. $\Phi^{*}$ commutes with rotations, so we may simultaneously diagonalize $\Phi^{*}$ and the rotation operator $-i(d / d \varphi)$. The eigenvalues of the rotation operator are of course the integers, with each eigenspace being $2 m$-dimensional. Specifically, the eigenspace corresponding to an integer $n$ is the span of the $2 m$ functions obtained by restricting $\exp (\operatorname{in} \varphi)$ to each of the $2 m$ circles in $X$. Operators on finite-dimensional spaces always have pure point spectra. Summing over $n$, we see that the spectrum of $\Phi^{*}$ is pure point.

On each $2 m$-dimensional subspace corresponding to the Fourier mode $\exp (\operatorname{in} \varphi)$, the action of $\Phi^{*}$ is described by a $2 m$ by $2 m$ matrix $E$, which we write as an $m \times m$ array 
of $2 \times 2$ matrices. Let $\theta=\tan ^{-1}(a / c)$ be the acute angle in our basic triangle. Consider the matrices

$$
\begin{aligned}
& A=\left(\begin{array}{cc}
0 & \exp [-i n(\theta+\pi / 2)] \\
\exp [i n(\theta+\pi / 2)] & 0
\end{array}\right) \\
& B=\left(\begin{array}{cc}
\exp (i n \theta)+\exp (i n(\theta+\pi)) & 2 \exp (-i n \theta) \\
2 \exp (i n \theta) & \exp (-i n \theta)+\exp (i n(-\theta+\pi))
\end{array}\right) .
\end{aligned}
$$

$A$ and $B$ describe the orientations of the five daughter tiles in terms of the orientation of the parent tile, as expressed in the $n$th representation of the rotation group $S O(2)$. Specifically, $A$ describes the daughter tile of hypotenuse $a$, while $B$ describes the four daughter tiles of hypotenuse $b / 2$. In each case the first column describes the daughters of a positively oriented tile, while the second column describes the daughters of a negatively oriented tile. See Fig. 9.

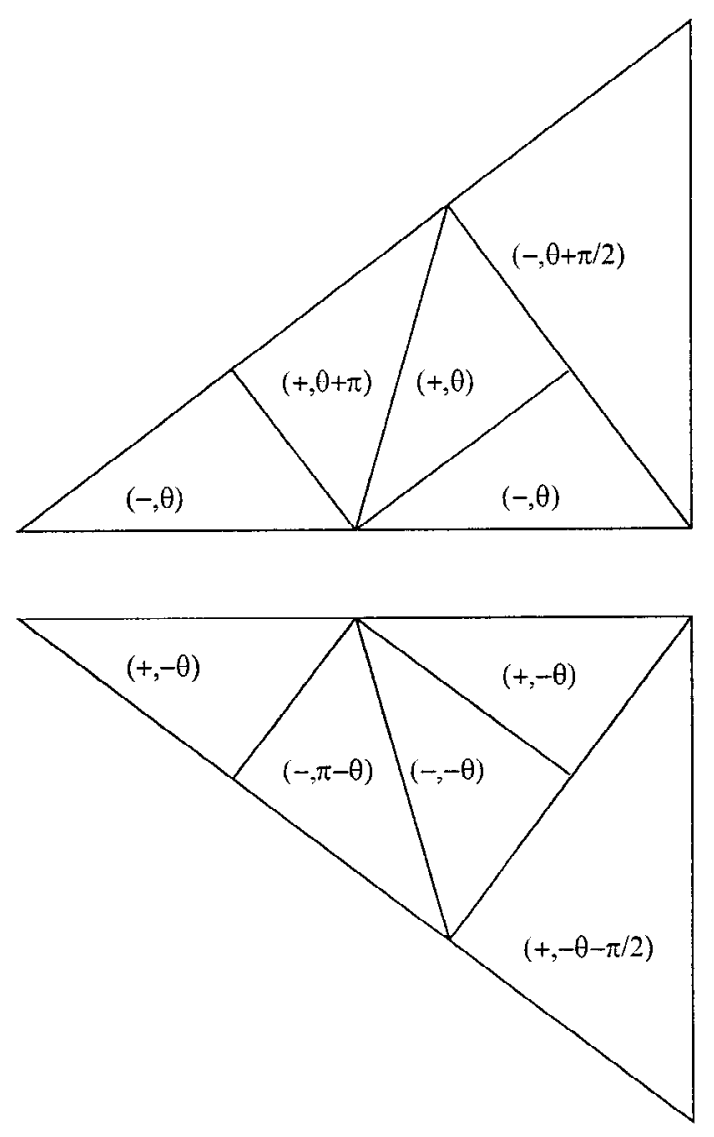

Fig. 9. Orientation of daughter tiles. 
Our matrix $E$ has the following matrix elements:

$$
E_{i j}= \begin{cases}1 & \text { if } j=i-1 \\ A & \text { if } i=1 \text { and } j=p \\ B & \text { if } i=1 \text { and } j=q \\ 0 & \text { otherwise. }\end{cases}
$$

Notice that $E$ is essentially the transpose of our population matrix $M$, with the daughters represented by the matrices $A$ and $B$ rather than the numbers 1 and 4 . We compute the eigenvalues and eigenvectors of $E$ by directly solving the equation $E \psi=\lambda \psi$.

Suppose $\psi_{m}=v$, where $v$ is a two-component vector. Each row but the first of $E \psi=\lambda \psi$ implies that $\psi_{k-1}=\lambda \psi_{k}$, and hence that $\psi_{k}=\lambda^{m-k} v$ for all $k$. The first row then says that

$$
\left(\lambda^{m}-\lambda^{m-q} B-\lambda^{m-p} A\right) v=0 .
$$

Taking the determinant of the matrix on the left-hand side, we obtain the characteristic polynomial of $E$,

$$
\begin{aligned}
p(\lambda)= & \lambda^{2 m}-\lambda^{2 m-q}(2 \cos (n \theta)+2 \cos (n \pi+n \theta))+\lambda^{2 m-2 q}(2 \cos (n \pi)-2) \\
& -\lambda^{2 m-2 p}-\lambda^{2 m-p-q}(4 \cos (n \pi / 2))
\end{aligned}
$$

First we consider $n=0$. If $p<q, p(\lambda)=\left(\lambda^{q}-\lambda^{q-p}-4\right)\left(\lambda^{p}+1\right) \lambda^{q-p}$. We recognize the first factor as the characteristic polynomial of $M$, with one root $r^{-2}$ and all other roots smaller in norm. The roots of the second and third factors have norm one and zero, respectively. The eigenvector corresponding to eigenvalue $r^{-2}$ is $\psi_{k}=r^{2 k}\left(\begin{array}{l}1 \\ 1\end{array}\right)$, i.e., $\zeta_{0}$ (up to scale).

We can understand the eigenvalues as follows. The roots of the first factor all correspond to $v=\left(\begin{array}{l}1 \\ 1\end{array}\right)$, and describe fluctuations in the numbers of tiles of various sizes, irrespective of orientation. This is the problem we previously studied in Theorem 6 . The unit and zero eigenvalues correspond to $v=\left(\begin{array}{c}1 \\ -1\end{array}\right)$. The zero eigenvalue has algebraic multiplicity $q-p$ and geometric multiplicity one. Since the basic subdivision produces equal numbers of positively and negatively oriented " $\mathrm{B}$ " tiles, after $q-p$ subdivisions there must be the same number of positively and negatively oriented tiles of each size $D_{p+1}, \ldots, D_{m}$. Since there is but one "A" daughter of each subdivided tile, the imbalance between positive and negative orientation in the larger sizes neither grows nor shrinks, but oscillates with period $2 p$. These correspond to the roots of $\lambda^{p}+1$.

If $p>q$, then $p(\lambda)$ factorizes as $p(\lambda)=\left(\lambda^{p}-1-4 \lambda^{p-q}\right)\left(\lambda^{p}+1\right)$. Again, the first factor is the characteristic polynomial of $M$, and governs the total number of tiles of each size, with all eigenvectors having $v=\left(\begin{array}{l}1 \\ 1\end{array}\right)$. The largest eigenvalue is $r^{-2}$, with eigenvector $\zeta_{0}$. The roots of $\left(\lambda^{p}+1\right)$ describe oscillations in the numbers of positively versus negatively oriented tiles and have $v=\left(\begin{array}{r}1 \\ -1\end{array}\right)$.

Next we consider $n$ odd. Then $\exp ($ in $\pi)=-1$, and $p(\lambda)$ simplifies to $\lambda^{2 m}-4 \lambda^{2 m-2 q}-$ $\lambda^{2 m-2 p}$. This is just the characteristic polynomial of $M$ applied to $\lambda^{2}$. By Theorem 6 , the largest roots have $\lambda^{2}=r^{-2}$ or $\lambda= \pm r^{-1}$.

Finally we consider $n$ even and nonzero. Then $p(\lambda)=\lambda^{2 m}-\lambda^{2 m-q}(4 \cos (n \theta))-$ $\lambda^{2 m-2 p}-\lambda^{2 m-p-q}(4 \cos (n \pi / 2))$. We show that all roots are smaller than $r^{-2}$ by the 
argument principle. Note that $\theta$ is an irrational multiple of $\pi$, so $\cos (n \theta) \neq \pm 1$. On the circle $|\lambda|=r^{-2}$ we have

$$
\begin{aligned}
\mid \lambda^{2 m-q}(4 & \cos (n \theta))|+| \lambda^{2 m-2 p}|+| \lambda^{2 m-p-q}(4 \cos (n \pi / 2)) \mid \\
& =\left|\lambda^{2 m}\right|\left(\frac{b^{2}|\cos (n \theta)|}{c^{2}}+\frac{a^{4}}{c^{4}}+\frac{a^{2} b^{2}}{c^{4}}\right) \\
& =\left|\lambda^{2 m}\right| \frac{a^{2}+b^{2}|\cos (n \theta)|}{c^{2}} \\
& <\left|\lambda^{2 m}\right| .
\end{aligned}
$$

Thus the $\lambda^{2 m}$ term of $p(\lambda)$ dominates on the circle $|\lambda|=r^{-2}$, so the winding of the argument of $p\left(r^{-2} \exp (i \alpha)\right)$, as $\alpha$ goes from zero to $2 \pi$, is $2 m$, and all $2 m$ eigenvalues of $E$ lie inside the circle of radius $r^{-2}$.

This completes the analysis of the spectrum of $\Phi^{*}$. Now we need only consider the decomposition into eigenvectors. Since $f$ is continuous, it has an absolutely summable Fourier series. So we may write $f(k, \Omega)=\sum_{n} c_{n} \psi_{k}^{(n)} \exp (\operatorname{in} \varphi)$, where the $c_{n}$ 's are absolutely summable and $\psi_{k}^{(n)}$ is a vector in $\mathbb{C}^{2 m}$ with no component larger than one. In particular, the partial sums converge uniformly to $f$. However, $\Psi_{k}$ is itself a sum of eigenvectors of $E^{|q-p|}$, so we may rewrite our sum as a sum of eigenfunctions of $\left(\Phi^{*}\right)^{|q-p|}$.

\section{Irrational Tilings}

In this section we consider tilings $\operatorname{Til}(z)$, with $z$ irrational. The analysis is formally similar to that of Section 4, except that we are now dealing with an infinite number of possible sizes. In place of the discrete-size parameter $k$ we introduce a continuous-size parameter $s$. In place of the discrete evolution operator $\Phi^{n}$ we introduce a one-parameter semigroup $e^{t L}$. Although the continuous case is technically more difficult than the discrete cases, the results are extremely similar. Indeed, if one has a sequence of rational numbers $p_{i} / q_{i}$ converging to the irrational number $z$, then the statistical properties of $\operatorname{Til}(z)$ may be obtained as limits of the corresponding properties of $\operatorname{Til}\left(p_{i} / q_{i}\right)$. (Note that the reverse does not hold. The statistics of a rational tiling cannot be obtained by taking a limit of irrational tilings.)

As always, we consider a basic right triangle $T_{0}$ with sides $a$ and $b$ and hypotenuse $c$. Let $\alpha=\ln (c / a), \beta=\ln (2 c / b)$, and assume that $z=\alpha / \beta$ is irrational. By a triangle of size $s$, we mean a triangle, similar to $T_{0}$, with hypotenuse $c e^{-s}$. Note that larger values of $s$ correspond to smaller triangles, just as in the rational case, where the size $D_{k}$ of triangles decreased with $k$. In our tiling the size parameter $s$ will take values in $[0, \mu)$, where $\mu=\max (\alpha, \beta)$.

We now describe a semigroup similar to $\Phi^{n}$. Let $S$ be a collection of tiles, all with size in $[0, \mu)$. Expand this collection by a linear factor $e^{t}$, resulting in triangles with sizes in $[-t, \mu-t)$. Then subdivide the largest triangle, subdivide the largest remaining triangle, and so on, until all triangles have nonnegative size parameter. By Lemmas 1 and 2, this occurs in a finite number of steps, and results in a collection of tiles with sizes in $[0, \mu)$. This collection is $e^{t L} S$. 
The semigroup $e^{t L}$ naturally acts on the distribution of sizes. The properties of this action are summarized by the following theorem, which should be compared with Theorem 6:

\section{Theorem 8.}

(1) The eigenvalues $\lambda$ of $L$ are the roots of $p(\lambda)=0$, where

$$
p(\lambda)=e^{\mu \lambda}-e^{(\mu-\alpha) \lambda}-4 e^{(\mu-\beta) \lambda} .
$$

There are no multiple eigenvalues.

(2) $\lambda=2$ is an eigenvalue. All other eigenvalues have real part strictly less than 2. If $\alpha<\beta$, all eigenvalues have real part greater or equal to the real root of $e^{\beta \lambda}+e^{(\beta-\alpha) \lambda}-4$, while if $\alpha>\beta$, all eigenvalues have real part greater or equal to the real root of $e^{\alpha \lambda}+4 e^{(\alpha-\beta) \lambda}-1$.

(3) The eigenfunction $\psi(s)$, for fixed eigenvalue $\lambda$, takes the form

$$
\psi(s)=e^{\lambda s}-e^{\lambda(s-\alpha)} h(s-\alpha)-4 e^{\lambda(s-\beta)} h(s-\beta),
$$

where $h(x)$ is the Heaviside function

$$
h(x)= \begin{cases}1 & \text { if } \quad x \geq 0 \\ 0 & \text { otherwise }\end{cases}
$$

(4) Given an interval I $\subset[0, \mu)$, the number of tiles with size in I is asymptotically a fraction

$$
\frac{1}{2 c^{2}} \int_{I} d s\left(a^{2} h(\alpha-s)+b^{2} h(\beta-s)\right) e^{2 s}
$$

of the total.

(5) Given an interval $I \subset[0, \mu)$, the area covered by tiles with size in I is asymptotically a fraction

$$
\frac{1}{a^{2} \alpha+b^{2} \beta} \int_{I} d s a^{2} h(\alpha-s)+b^{2} h(\beta-s)
$$

of the total.

Corollary. Let $T$ be a tile of size s. Let $N_{t}(s)$ be the number of tiles in $e^{t L}(T)$. Then

$$
\lim _{t \rightarrow \infty} e^{-2 t} N_{t}(s)=\frac{2 c^{2} e^{-2 s}}{a^{2} \alpha+b^{2} \beta} .
$$

Proof of the Corollary. From the distribution (5.4), we compute the average area per tile to be $a b\left(a^{2} \alpha+b^{2} \beta\right) / 4 c^{2}$. A tile of size $s$, rescaled by a factor $e^{t}$, has area $a b e^{2 t-2 s} / 2$. Dividing by the area per unit tile we obtain (5.6). 
Proof of Theorem 8. For $0<t<\min (\alpha, \beta,|\alpha-\beta|)$, the action of $e^{t L}$ on the population distribution functions is

$$
\left(e^{t L} \psi\right)(s)= \begin{cases}\psi(s+t)+4 \psi(s+t-\beta) & \text { if } \quad s \in[\beta-t, \beta) \\ \psi(s+t)+\psi(s+t-\alpha) & \text { if } \quad s \in[\alpha-t, \alpha) \\ \psi(s+t) & \text { all other } \quad s \in[0, \mu)\end{cases}
$$

where $\psi(s)$ and $e^{t L} \psi(s)$ are understood to be zero for $s \geq \mu$ or $s<0$. $e^{t L}$ acts continuously on functions that are continuous away from $0, \alpha, \beta$, and for which the oscillations at $\alpha$ and $\beta$ are given by

$$
\psi\left(\alpha^{-}\right)-\psi\left(\alpha^{+}\right)=f(0) ; \quad \psi\left(\beta^{-}\right)-\psi\left(\beta^{+}\right)=4 f(0) .
$$

Setting $e^{t L} \psi=e^{t \lambda} \psi$ we see that the eigenfunction $\psi(s)$ must equal $e^{\lambda s}$ times a piecewise constant function with discontinuities at $0, \alpha, \beta$. Applying the boundary conditions (5.8), we obtain the eigenfunction (5.2). For $s>\mu, \psi(s)$ then equals $e^{(s-\mu) \lambda}$ times $p(\lambda)$. The vanishing of $\psi(s)$ for $s>\mu$ is equivalent to the eigenvalue equation $p(\lambda)=0$. Thus eigenfunctions satisfying the boundary conditions are in 1-1 correspondence with roots of $p(\lambda)$, with the eigenfunctions given by (5.2).

Suppose $\alpha<\beta$, in which case $p$ takes the form

$$
p(\lambda)=e^{\beta \lambda}-e^{(\beta-\alpha) \lambda}-4 .
$$

$\lambda=2$ is a root, since $p(2)=e^{2 \beta}-e^{2(\beta-\alpha)}-4=4 c^{2} / b^{2}-4 a^{2} / b^{2}-4=0$. This is the only real root, insofar as $p(\lambda)$ is an increasing function of $\lambda$ for $\lambda>0$, and $p(\lambda)$ is negative for $\lambda \leq 0$. In particular, $p(\lambda)<0$ implies that $\lambda<2$.

Now consider complex roots $\lambda=\lambda_{R}+i \lambda_{I}$. If $\lambda_{I} \neq 0, e^{\beta \lambda}$ and $e^{(\beta-\alpha) \lambda}$ cannot both be real, insofar as $\beta$ is not a rational multiple of $\beta-\alpha$. Thus $\left|e^{\beta \lambda}\right|,\left|e^{(\beta-\alpha) \lambda}\right|$, and 4 satisfy a strict triangle inequality. In particular,

$$
0>\left|e^{\beta \lambda}\right|-\left|e^{(\beta-\alpha) \lambda}\right|-4=p\left(\lambda_{R}\right)
$$

so $\lambda_{R}<2$. Also,

$$
0<\left|e^{\beta \lambda}\right|+\left|e^{(\beta-\alpha) \lambda}\right|-4=e^{\beta \lambda_{R}}+e^{(\beta-\alpha) \lambda_{R}}-4,
$$

so $\lambda_{R}$ is greater than the real root of $e^{\beta \lambda}+e^{(\beta-\alpha) \lambda}-4$.

Now we exclude the possibility of multiple roots. A multiple root would require $p(\lambda)=p^{\prime}(\lambda)=0$. Suppose $0=p^{\prime}(\lambda)=\beta e^{\beta \lambda}+(\beta-\alpha) e^{(\beta-\alpha) \lambda}$. Then $e^{\beta \lambda}$ and $e^{(\beta-\alpha) \lambda}$ must have the same phase, and their difference must also have that phase. However, if $\lambda$ is not real, $e^{\beta \lambda}$ and $e^{(\beta-\alpha) \lambda}$ cannot both be real, so their difference is not real, so their difference is not four. Thus $p^{\prime}(\lambda)=0$ implies that $p(\lambda) \neq 0$, and there are no multiple roots away from the real axis. On the real axis, the only root is $\lambda=2$, and we have already seen that $p^{\prime}(2)$ is positive, not zero. 
This establishes statements (1)-(3) for the case $\alpha<\beta$. The argument for $\alpha>\beta$ is almost identical, and is not repeated.

Now suppose that we initially have a population distribution that is a linear combination of the eigenfunctions $\psi_{\lambda}$. Applying $e^{t L}$ to the system, the total area grows as $e^{2 t}$, so the number of tiles is bounded, both above and below, by a multiple of $e^{2 t}$. Applying $e^{t L}$ and dividing by the number of tiles damps out all the modes with eigenvalue less than two, i.e., all eigenvectors other than $\psi_{2}$. In this case, the final distribution of sizes approaches a multiple of $\psi_{2}$, and statements (4) and (5) of the theorem follow.

Unfortunately, we cannot a priori assume that the initial condition is a linear combination of eigenfunctions of $L$, or that a test function is a linear combination of eigenfunctions of the dual operator $L^{*} . e^{t L}$ and $e^{t L^{*}}$ are neither finite-rank operators nor self-adjoint operators on a Hilbert space, so standard theorems about the completeness of a basis is eigenfunctions cannot be applied. In principle it is possible for a test function $f$ to have the property that $e^{t\left(L^{*}-2\right)} f$ does not converge at all. We must show that, when $f=\chi_{I}$ (the characteristic function of an interval $I), e^{t\left(L^{*}-2\right)} f$ does converge.

Lemma 10. Given an interval $I \in[0, \mu)$. The fraction of the area of $e^{t L}\left(T_{0}\right)$ covered by tiles with size in I approaches a limit as $t \rightarrow \infty$.

Given this lemma, it follows that the distribution of area of $e^{t L}(S)$, for any collection of tiles $S$, approaches a limit, from which it follows that the distribution of population of $e^{t L}(S)$ also approaches a limit. Since these limits are invariant under further evolution, and since the total area is proportional to $e^{2 t}$, these limits must correspond to the $\lambda=2$ eigenvector of $L$, hence must take the form (5.4) and (5.5).

Proof of Lemma 10. Assume $\alpha<\beta$; the other case is similar. Let $F_{I}(t)$ be the fraction of area of $e^{t L}\left(T_{0}\right)$ covered by tiles with size in $I$. Given an $\varepsilon>0$, we show how to compute a number such that, for all $t$ sufficiently large, $F_{I}(t)$ is within $\varepsilon$ of this number. Since this can be done for any $\varepsilon, \lim _{t \rightarrow \infty} F_{I}(t)$ must exist.

It suffices to show that the eventual fraction in an interval of size $\Delta$, entirely in $[0, \alpha)$ or in $[\alpha, \beta)$, and with $\Delta$ sufficiently small, can be estimated to within $O\left(\Delta^{2}\right)$. Any larger interval can be broken up into a finite number of such small pieces, such that $\sum$ errors $<\varepsilon$. So we fix an interval $I$, centered at $s_{0}$, with width $\Delta$.

The strategy is this: We begin with an exact expression for the fraction of area of $e^{t L}\left(T_{0}\right)$ represented by tiles of size $s$. We sum this over $s \in I$ to get an exact formula for $F_{I}(t)$. By taking certain limits and replacing certain sums with integrals, we obtain an expression that is independent of $t$. In the process we introduce two types of errors. One type can be made arbitrarily small by requiring $t$ to be sufficiently large. The other type is $O\left(\Delta^{2}\right)$.

How many triangles of size $s$ appear in $e^{t L}\left(T_{0}\right)$ ? That depends on whether $s+t$ can be written as $n_{1} \alpha+n_{2} \beta$ for nonnegative $n_{1}$ and $n_{2}$. If $s+t=n_{1} \alpha+n_{2} \beta$, then a triangle of size $s$ may be obtained by taking a triangle of size $-t$, subdividing it, picking a daughter, subdividing it, picking a daughter, and so on for $n_{1}+n_{2}$ subdivisions, with the descent involving $n_{1}$ daughters of type $A$ and $n_{2}$ daughters of type $B$. If $s \geq \alpha$, then the last daughter must be of type $B$, or else after $n_{1}+n_{2}-1$ steps we would have already obtained a tile of size in $[0, \beta)$, and would not have made the final subdivision. If $s<\alpha$ 
there is no such constraint. We thus have

number of tiles of size $s$

$$
= \begin{cases}\left(\begin{array}{c}
n_{1}+n_{2} \\
n_{1}
\end{array}\right) 4^{n_{2}} & \text { if } \quad s+t=n_{1} \alpha+n_{2} \beta \quad \text { and } \quad s \in[0, \alpha) ; \\
\left(\begin{array}{c}
n_{1}+n_{2}-1 \\
n_{1}
\end{array}\right) 4^{n_{2}} & \text { if } \quad s+t=n_{1} \alpha+n_{2} \beta \quad \text { and } \quad s \in[\alpha, \beta) ; \\
0 & \text { otherwise. }\end{cases}
$$

Since at each division a fraction $a^{2} / c^{2}$ of the area goes into the $A$ daughter, while a fraction $b^{2} / c^{2}$ goes into the $B$ daughters, then the fraction of the total area represented by tiles of size $s$ is

$$
F_{s}(t)= \begin{cases}\left(\begin{array}{c}
n_{1}+n_{2} \\
n_{1}
\end{array}\right)\left(\frac{a^{2}}{c^{2}}\right)^{n_{1}}\left(\frac{b^{2}}{c^{2}}\right)^{n_{2}} & \text { if } s+t=n_{1} \alpha+n_{2} \beta \\
\left(\begin{array}{c}
n_{1}+n_{2}-1 \\
n_{1}
\end{array}\right)\left(\frac{a^{2}}{c^{2}}\right)^{n_{1}}\left(\frac{b^{2}}{c^{2}}\right)^{n_{2}} & \text { and } s \in[0, \alpha) ; \\
0 & \text { if } s+t=n_{1} \alpha+n_{2} \beta \\
& \text { and } s \in[\alpha, \beta) ; \\
\text { otherwise. }\end{cases}
$$

Note that $n_{2}=\left(s+t-\alpha n_{1}\right) / \beta$. Now let

$$
f(s, t, n)= \begin{cases}\left(\begin{array}{c}
(s+t+n(\beta-\alpha)) / \beta \\
n
\end{array}\right)\left(\frac{a^{2}}{c^{2}}\right)^{n}\left(\frac{b^{2}}{c^{2}}\right)^{(s+t-\alpha n) / \beta} & \text { if } s \in[0, \alpha) ; \\
\left(\begin{array}{c}
(s+t+n(\beta-\alpha)-\beta) / \beta \\
n_{1}
\end{array}\right)\left(\frac{a^{2}}{c^{2}}\right)^{n}\left(\frac{b^{2}}{c^{2}}\right)^{(s+t-\alpha n) / \beta} & \text { if } s \in[\alpha, \beta),\end{cases}
$$

and let $\delta_{p}$ be the periodic $\delta$-function

$$
\delta_{p}(x)=\sum_{n \in \mathbb{Z}} \delta(x-n) .
$$

Note that $f(s, t, n)$ is well-defined even when $(s+t+n(\beta-\alpha)) / \beta$ is not an integer. For $t$ large, $f(s, t, n)$ is a slowly varying function of $s$ and $n$.

We then compute, exactly,

$$
F_{I}(t)=\sum_{s \in I} F_{s}(t)=\sum_{n=0}^{[t / \alpha]} \int_{I} f(s, t, n) \delta_{p}((s+t-n \alpha) / \beta) .
$$

Next we approximate, by replacing $f(s, t, n)$ by $f\left(s_{0}, t, n\right)$, where $s_{0}$ is the midpoint of $I$. This introduces an error that is a fraction $O(\Delta)$ of the total. Since the total will turn out to be $O(\Delta)$, the error introduced is $O\left(\Delta^{2}\right)$. We thus have

$$
F_{I}(t)=\sum_{n=0}^{[t / \alpha]} f\left(s_{0}, t, n\right) \int_{I} \delta_{p}((s+t-n \alpha) / \beta)+O\left(\Delta^{2}\right) .
$$


Next we use the fact that multiples of an irrational number are uniformly distributed on $\mathbb{R} / \mathbb{Z}$. For any desired degree of accuracy, an $N$ can be found such that, for any $N$ consecutive integers $n_{i}, \int_{I} \delta_{p}\left(\left(s+t-n_{i} \alpha\right) / \beta\right)$ equals one a fraction $\Delta / \beta$ of the time (to within the allowed error), and zero the rest of the time. If we restrict ourselves to $t$ so large that $f\left(s_{0}, t, n\right)$ is nearly constant as $n$ varies over intervals of size $N$, then

$$
\sum_{n=0}^{[t / \alpha]} f\left(s_{0}, t, n\right) \int_{I} \delta_{p}((s+t-n \alpha) / \beta) \approx(\Delta / \beta) \sum_{n=0}^{[t / \alpha]} f\left(s_{0}, t, n\right) .
$$

Finally, the $t \rightarrow \infty$ limit of the resulting sum can be expressed as an integral, and yields a nonzero number.

We now turn, as in Section 4, to the joint distribution of sizes and orientation. We parametrize the space of possible orientations of a single size, as in Section 4 , by two copies of the unit circle, with Haar measure $d \Omega$. (See Fig. 8.) The space of all possible tiles up to translation, which we denote $X$, is two copies of $S^{1} \times[0, \mu)$.

Let $f$ be a function on $X$. Given a collection $S$ of tiles, let $\langle f, S\rangle$ be the average value of $f$ on the individual tiles in $S$, where each tile is given equal weight. Let $\langle f, S\rangle^{\prime}$ be the average value of $f$ on the individual tiles in $S$, where each tile is weighted proportionally to its area. Let $(f, S)$ be the sum of $f$ on the individual tiles of $S$. We define a semigroup $e^{t L^{*}}$ acting on functions on $X$ by

$$
\left(e^{t L^{*}} f, S\right)=\left(f, e^{t L}(S)\right) .
$$

Theorem 9. Assume a tiling Til(z), with $z$ irrational and with $\theta=\tan ^{-1}(a / b)$ an irrational multiple of $\pi$. Let $f$ be a continuous function on $X$, and let $\left\{S_{n}\right\}$ be a sequence of supertiles of increasing size. Let $d v=e^{2 s}\left(a^{2} h(\alpha-s)+b^{2} h(\beta-s)\right) d \Omega d s / 2 c^{2}$ and $d \rho=2 c^{2} e^{-2 s} d v /\left(a^{2} \alpha+b^{2} \beta\right)$ be measures on $X$, where $h$ is the Heaviside function (5.3). Then

$$
\begin{aligned}
& \lim _{n \rightarrow \infty}\left\langle f, S_{n}\right\rangle=\int_{X} f d \nu, \\
& \lim _{n \rightarrow \infty}\left\langle f, S_{n}\right\rangle^{\prime}=\int_{X} f d \rho .
\end{aligned}
$$

These limits also apply if $f$ is the characteristic function of a rectangle in $X$.

Remark. The measures $d v$ and $d \rho$ are closely related to the integrands in (5.4) and (5.5), respectively. Theorem 9 states that the joint distribution of sizes and orientations is a product: the size distribution previously found in Theorem 8 times a uniform distribution of orientations.

Proof. The proof is extremely similar to the proof of Theorem 7. As in that case, it is sufficient to establish (5.20a) for an arbitrary continuous function $f$. Such a function can be written as an absolutely convergent sum of Fourier modes (with respect to rotations). The coefficient of each mode is a $\mathbb{C}^{2}$-valued function of $s$. The operator $e^{t L^{*}}$ commutes with rotation, and so acts separately on each Fourier mode. 
On the $n$th Fourier mode, $e^{t L^{*}}$, for $t$ small, acts as follows:

$$
\left(e^{t L^{*}} f\right)(s)= \begin{cases}A \psi(s-t+\alpha)+B \psi(s-t+\beta) & \text { if } \quad s \in[0, t) \\ \psi(s-t) & \text { all other } \quad s \in[0, \mu),\end{cases}
$$

where the matrices $A$ and $B$ are, as in Section 4,

$$
\begin{gathered}
A=\left(\begin{array}{cc}
0 & \exp [-i n(\theta+\pi / 2)] \\
\exp [i n(\theta+\pi / 2)] & 0
\end{array}\right), \\
B=\left(\begin{array}{cc}
\exp (i n \theta)+\exp (i n(\theta+\pi)) & 2 \exp (-i n \theta) \\
2 \exp (i n \theta) & \exp (-i n \theta)+\exp (i n(-\theta+\pi))
\end{array}\right) .
\end{gathered}
$$

The zeroth Fourier mode decouples into $\left(\begin{array}{l}1 \\ 1\end{array}\right)$ and $\left(\begin{array}{r}1 \\ -1\end{array}\right)$ components. The $\left(\begin{array}{l}1 \\ 1\end{array}\right)$ component is the distribution of sizes regardless of orientation, and its asymptotic behavior was already computed in Theorem 8 . We must show that the $n=0\left(\begin{array}{r}1 \\ -1\end{array}\right)$ component, and all the Fourier modes with $n \neq 0$, grow strictly slower than the size of the system, and so represent a decreasing fraction of the system.

We will control the $L^{1}$ norms of the unwanted Fourier modes. To do this we need the $L^{1}$ norms of the matrices $A$ and $B$, and various products of $A$ and $B$. The $L^{1}$ norm of a matrix is maximum, over all columns, of the sum of the absolute values of the entries in that column. One can get a bound on the growth of the $L^{1}$ norm of a mode by the mode with its absolute value, and replacing the matrices $A$ and $B$ by their norms.

For $n=0, B\left(\begin{array}{c}1 \\ -1\end{array}\right)=0$. With $B=0$ it is as if there is only one daughter per division, hence the $L^{1}$ norm of the $\left(\begin{array}{c}1 \\ -1\end{array}\right)$ mode at time $t$ is bounded by the $L^{1}$ norm of the mode at time zero. Hence, as a fraction of the system, this mode shrinks like $e^{-2 s}$.

Next we consider $n$ odd, for which the diagonal terms in $B$ vanish. The sum of the absolute values of the entries of each column of $B$ equals two. This is as if, at each subdivision, only two daughter $B$ tiles are produced, instead of four. To put it another way, at each subdivision a fraction $b^{2} / 2 c^{2}$ of the area is lost. Since each piece of a tile of size $-t$ must be divided at least $t / \beta$ times, this means that the $L^{1}$ norm of the $n$th mode, for $n$ odd, can grow no faster than $e^{2 t}\left[1-\left(b^{2} / 2 c^{2}\right)\right]^{t / \beta}$ and so, as a fraction of the system, goes to zero.

Finally we consider $n$ even but nonzero. Here the column sums of $A$ and $B$ are the same as in the $n=0$ case, namely one and four, respectively. However, the $L^{1}$ norms of various products of $A$ and $B$ are smaller that in the $n=0$ mode. For example, $B^{2}=4 \cos (n \theta) B$ has norm $16|\cos (n \theta)|$, which is strictly smaller than 16 . The norm of $B A B$ is also $16|\cos (n \theta)|$. Indeed, the only words in $A$ and $B$ which have norms as large as in the $n=0$ case are $A^{m}$ and $A^{m_{1}} B A^{m_{2}}$. Since the expansion of $e^{t L^{*}} f$, for $t>\beta$, involves expressions such as $B^{2}$, the growth of the $L^{1}$ norm of $n$th Fourier mode is bounded by an exponent strictly less than two. As a fraction of the system, the $n$th mode goes to zero.

Remark. The spectrum of $e^{t L^{*}}$ may be obtained exactly as in Section 4. In seeking eigenvectors, (4.20) is replaced by

$$
\left(e^{\mu \lambda}-e^{(\mu-\beta) \lambda} B-e^{(\mu-\alpha) \lambda} A\right) v=0 .
$$

Subsequent analysis may be repeated word for word, replacing $\lambda$ by $e^{\lambda}, p$ by $\alpha, q$ by $\beta$, $m$ by $\mu, \psi_{k}$ by $\psi(s)$, and $r^{k}$ by $e^{-s}$. 


\section{Two Exceptional Tilings- $\operatorname{Til}(1 / 3)$ and $\operatorname{Til}(2)$}

We saw in Section 4 how the population statistics of a rational tiling $\operatorname{Til}(p / q)$ depends on $p$ and $q$. If $q>1$, the second eigenvalue of the population matrix is greater than one, and fluctuations increase with subdivision. This leads to phenomena such as slippage along fault lines and a failure to be globally edge-to-edge. A typical example, Til( $1 / 2)$, was studied in Section 3.

In this section we study examples of the remaining cases. We study Til(2) as an example of a Til $(p / 1)$ tiling. In all such tilings, the second eigenvalue of the population matrix is less than one. We shall see how, in the case of Til(2), this prevents slippage along fault lines.

Finally, we consider $\operatorname{Til}(1 / 3)$, the only rational tiling to exhibit only a finite number of orientations of each size of tile. As in all cases with $q>1$, there is an eigenvalue greater than one in the problem. The fluctuations governed by this eigenvalue are enough to force Til $(1 / 3)$ to be nonperiodic. However, because of rational relations between the lengths of certain edges, the tiles in Til(1/3) meet in only a finite number of ways.

We begin with $\operatorname{Til}(2)$. Til(2) is based on the right triangle with $a=\sqrt{5}-2 \approx 0.2361$, $b=2 \sqrt{\sqrt{5}-2} \approx 0.9717, c=1$. Several iterations of the subdivision are shown in Fig. 10. An essential feature of $\operatorname{Til}(2)$ is

Theorem 10. The triangles in the tiling Til(2) meet in only a finite number of ways.

Proof. The proof is essentially in two steps. First we show that the slippage along the primary fault line is bounded. Then we show that slippage along a fault line is the only

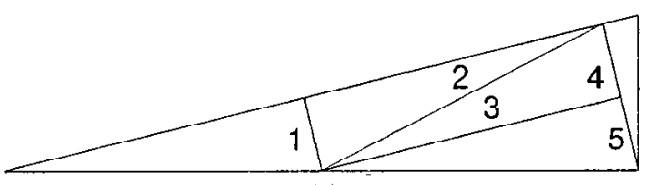

(a)

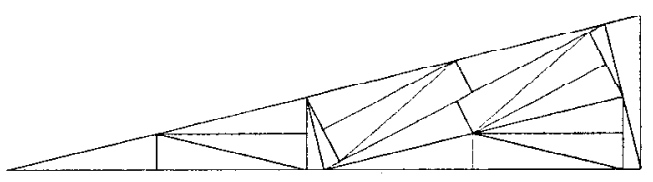

(b)

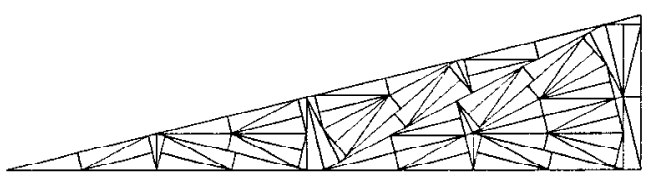

(c)

Fig. 10. Three stages of subdivision for Til(2). 
means by which tiles in a rational tiling can meet in an infinite number of ways, and that all fault lines are similar to the primary fault line.

To examine what happens along the primary fault line, we consider the boundary of $T_{2 n}$, the $2 n$th subdivision of the basic triangle. Note that the hypotenuse and short leg of $T_{2}$ consist only of hypotenuses and short legs of big triangles. Applying the subdivision again, we get that the hypotenuse and short leg of $T_{4}$ also consists only of hypotenuses and short legs of big triangles. Similarly for all $T_{2 n}$.

As in the proof of Theorem 5, we consider the evolution of these legs and hypotenuses as a one-dimensional substitution system in its own right. Let $\sigma$ denote the effect of subdividing the basic triangle twice. Under $\sigma$, each hypotenuse (denoted $H$ ) is replaced by four hypotenuses and a short leg (denoted $S$ ), while each short leg is replaced by a hypotenuse. That is, the one-dimensional population matrix is

$$
M=\left(\begin{array}{ll}
4 & 1 \\
1 & 0
\end{array}\right)
$$

with eigenvalues $\lambda_{ \pm}=2 \pm \sqrt{5}$ and eigenvectors

$$
v_{ \pm}=\left(\begin{array}{c}
\lambda_{ \pm} \\
1
\end{array}\right)
$$

Now let $H_{n}$ and $S_{n}$ be the number of hypotenuses and short legs in $\sigma^{n}(H)$, and let $H_{n}^{\prime}$ and $S_{n}^{\prime}$ be the number of hypotenuses and short legs in $\sigma^{n}(S)$. By expanding $\left(\begin{array}{l}0 \\ 1\end{array}\right)$ and $\left(\begin{array}{l}1 \\ 0\end{array}\right)$ in terms of $v_{ \pm}$, it is easy to see that

$$
(\sqrt{5}-2) H_{n}-S_{n}=-(2-\sqrt{5})^{n+1} ; \quad(\sqrt{5}-2) H_{n}^{\prime}-S_{n}^{\prime}=-(2-\sqrt{5})^{n} .
$$

Next we measure slippage. Let $P$ and $R$ be the endpoints of a hypotenuse, as in Fig. 7, and let $E$ be any intermediate point, not necessarily the midpoint. Let $f_{n}(E)$ be the number of complete short legs, between $P$ and $E$, in $\sigma^{n}(P R)$, minus the number of short legs between $P$ and $E$ in $\sigma^{n}(R P)$. As in the proof of Theorem 5, $f_{n}(E)$ measures the extent to which the two tiles of $T_{2 n+2}$ that meet at $E$ are offset.

Lemma 11. $\left|f_{n}(E)\right|<5$.

Proof. Let $v_{0}=P$, and let $v_{k}$ be the vertex of $\sigma^{k}(P R)$, between $P$ and $E$, that is closest to $E$. Note that, in $\sigma^{k}(P R)$, there are at most four hypotenuses and at most one short leg between $v_{k-1}$ and $v_{k}$, since the interval $v_{k-1} v_{k}$ was only part of a hypotenuse or short leg in $\sigma^{k-1}(P R)$. By $(6.2),(\sqrt{5}-2)$ times the number of hypotenuses in $\sigma^{n}(P R)$ between $v_{k-1}$ and $v_{k}$, minus the number of short legs, is bounded in absolute value by $(\sqrt{5}-2)^{n-k}$. Summing over $k$, we get that $(\sqrt{5}-2)$ times the number of complete hypotenuses between $P$ and $E$ in $\sigma^{n}(P R)$, minus the number of short legs, is bounded in absolute value by $\sum_{i=0}^{\infty}(\sqrt{5}-2)^{i}<2$. A similar bound applies to the number of hypotenuses and legs in $\sigma^{n}(R P)$. Thus the surplus of short legs on one side of $P E$ relative to the other, plus $(\sqrt{5}-2)$ times the deficit of hypotenuses, is bounded by $2+2=4$. Since a surplus of short legs implies a deficit of hypotenuses, the surplus of short legs is itself bounded by four. 
We return to the proof of Theorem 10. Lemma 11 limits the number of ways for two tiles to meet across a fault line. Suppose two tiles $t_{1}$ and $t_{2}$ meet across a fault line $P R$, modeled on $\sigma^{n}(H)$. Let $E$ be a point on their common edge. The distance from the vertex of $t_{1}$ closest to $P$ to the vertex of $t_{2}$ closest to $P$ is either $\left|f_{n}(E)\right|(\sqrt{5}-$ 2) or $1-\left|f_{n}(E)\right|(\sqrt{5}-2)$. Since $\left|f_{n}\right|$ is at most four, this means there are only a finite number of ways for two triangles to meet across such fault lines. Now $\sigma^{n}(H)$ is the result of subdividing the basic triangle an even number of times. However, since subdivision is deterministic, having only a finite number of distinct configurations in the even subdivisions implies that there are only a finite number of distinct configurations in the odd subdivisions, and thus a finite number of configurations in all.

To complete the proof of Theorem 10, we must show that every pair of adjacent triangles either meets full-face to full-face, or meets across a fault line based on successive subdivisions of a hypotenuse. Consider two tiles, $t_{1}$ and $t_{2}$, that meet. Let $k$ be the smallest integer such that both tiles lie in the same supertile $S$ of order $k$. Since $t_{1}$ and $t_{2}$ do not meet in a supertile of order $k-1, t_{1}$ and $t_{2}$ must meet across one of the five lines of the first subdivision of $S$. See Fig. 10(a). There is a local reflection symmetry across edges 1 and 3 , so if $t_{1}$ and $t_{2}$ meet across these edges they must meed full-face to full-face. Edge 2 is the primary fault line. Further division (see Fig. 10(c)) shows that there is local reflection symmetry across edge 4 , while edge 5 is a hypotenuse-based fault line, as considered above.

We now turn to the tiling Til(1/3). By Theorem 4, Til(1/3) is the only tiling in our construction to have both a finite number of sizes of tiles, each of which appears only in a finite number of orientations. Til (1/3) is based on an isosceles right triangle. Although the two legs have the same length, we distinguish between the two, calling the " $b$ " side "long" and the " $a$ " side "short," in analogy to the tilings with $b>a$. In subdividing we must specify which legs of the daughter tiles are labeled "long" and "short." This is shown in Fig. 11, and several further subdivisions are shown in Fig. 12.

Theorem 11. The tiling Til(1/3) is nonperiodic. The tiles meet in only a finite number of ways.

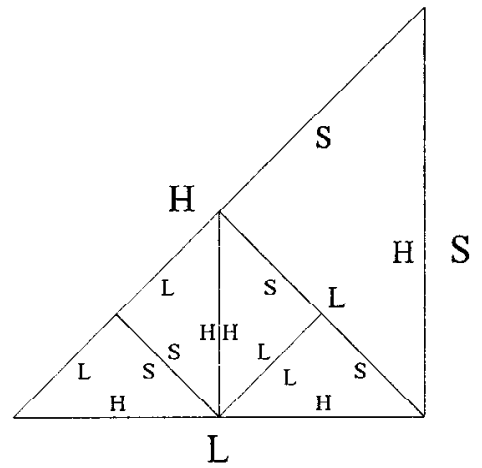

Fig. 11. "Long" and "short" edges in Til(1/3). 

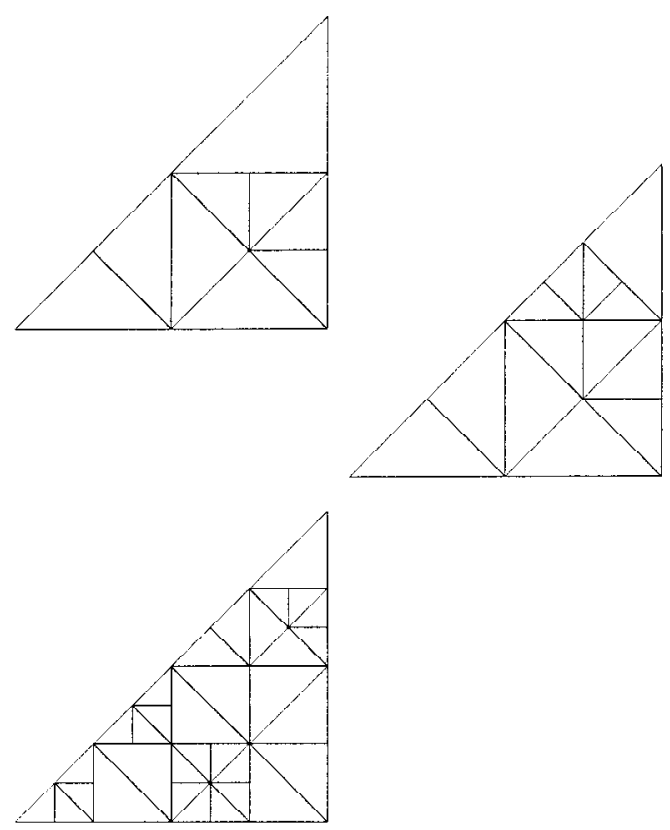

Fig. 12. Three stages of subdivision for Til(1/3).

Proof. As usual, we consider the one-dimensional substitution scheme induced on the edges by two-fold substitution. The $2 n$th subdivision of the hypotenuse consists of some hypotenuses of large triangles (denoted $H$ ), some long legs of medium-sized triangles $(L)$, and some hypotenuses of small triangles $(h)$. Let $\sigma$ denote the action of two subdivisions. $\sigma$ takes each $H$ to an $H$ and two $L$ 's, each $L$ to two $h$ 's, and each $h$ to an $H$. The population matrix is

$$
M=\left(\begin{array}{lll}
1 & 0 & 1 \\
2 & 0 & 0 \\
0 & 2 & 0
\end{array}\right)
$$

with eigenvalues 2 and $(-1 \pm \sqrt{-7}) / 2$, and with the eigenvector $\left(\begin{array}{l}1 \\ 1 \\ 1\end{array}\right)$ corresponding to eigenvalue 2. Note that the complex eigenvalues have magnitude $\sqrt{2}$.

Asymptotically, the three types of edges appear in a ratio of $1: 1: 1$. However, since the initial condition $\left(\begin{array}{l}1 \\ 0 \\ 0\end{array}\right)$ is not an eigenvector, and since all eigenvalues are greater than one (in magnitude), the difference in number between $H$ 's and $L$ 's, or $L$ 's and $h$ 's, will grow exponentially with time. That is, while the total population grows as $2^{n}$, the fluctuations grow as $\sqrt{2}^{n}$.

If $\operatorname{Til}(1 / 3)$ were periodic, a long line of the form $\sigma^{n}(H)$ would consist of several periods, plus a remainder at each end. Each period would have $H$ 's, $L$ 's, and $h$ 's in exactly a 1:1:1 ratio, so only the partial periods at each end could contribute to the difference in population between $H$ and $L$. Thus the population difference would remain bounded as $n \rightarrow \infty$. Since this difference is unbounded, Til(1/3) cannot be periodic. 
Finally we note that the length of $h$ is the same as that of $L$, and half that of $H$. This simple ratio of lengths means that slippage along the fault line has no effect on the number of ways triangles can meet. If two tiles meet across $\sigma^{n}(H)$, either they have a vertex in common or their closest vertices are separated by the length of $h$. Thus there are only a finite number of ways for two tiles to meet across a fault line. By the same argument as in the proof of Theorem 10, this implies that there are only a finite number of ways for triangles to meet at all.

\section{Conclusions}

We have constructed a family of substitution systems, indexed by the parameter $z=$ $\ln (\sin (\theta)) / \ln [\cos (\theta) / 2]$, where $\theta$ is an angle in the basic triangular tile. We have established the following properties.

(1) The tilings generated by these substitutions are all nonperiodic.

(2) The tilings have well-defined limiting distributions of size and orientation. If $\theta / \pi$ is irrational, this distribution is rotationally invariant. In Radin's terminology, the tilings have "statistical rotational symmetry." The form of the joint distribution of size and orientation suggests that the tiling has a purely absolutely continuous spectrum.

(3) The tilings with rational $z$ all satisfy the hypotheses of Goodman-Strauss's theorem, implying that they can be forced through local matching rules.

(4) The rational tilings $\operatorname{Til}(p / q)$, with $q>1$, have statistical fluctuations that grow with iterations of the substitution rule (although they grow slower than the size of the system). In Til(1/2) these fluctuations force triangles to meet in an infinite number of distinct ways. We conjecture that this infinite diversity of local behavior is a property of all rational tilings $\operatorname{Til}(p / q)$ with $q>1$ and $p / q \neq 1 / 3$.

(5) In the rational tilings $\operatorname{Til}(p / 1)$ the eigenvalues that control fluctuations are all less than one. In Til(2) this forces the tiles to meet in only a finite number of local patterns. We conjecture that this is a property of all tilings $\operatorname{Til}(p / 1)$.

\section{Acknowledgments}

It is a pleasure to thank Chaim Goodman-Strauss, Yoram Last, Tom Mrowka, Johan Råde, Charles Radin, and Felipe Voloch for useful discussions, and Steven Janowsky for assistance with computer graphics.

\section{References}

[CFS] I. Cornfeld, S. Fomin, and, Ya. Sinai, Ergodic Theory, Springer-Verlag, New York, 1992

[G] C. Goodman-Strauss, Matching rules and substitution tilings, Preprint, 1996.

[R1] C. Radin, The pinwheel tilings of the plane, Ann. of Math. 139 (1994), 661-702.

[R2] C. Radin, Space tilings and substitutions, Geom. Dedicata 55 (1995), 257-264.

[R3] C. Radin, Symmetry and tilings, Notices Amer. Math. Soc. 42 (1995), 26-31.

[S] M. Senechal, Quasicrystals and Geometry, Cambridge University Press, Cambridge, 1995.

Received April 9, 1996, and in revised form September 16, 1996. 